\title{
Characterization of the Ketosynthase and Acyl Carrier Protein Domains at the Lnml Nonribosomal Peptide Synthetase-Polyketide Synthase Interface for Leinamycin Biosynthesis
}

Yong Huang, ${ }^{\dagger, ~}$ Gong-Li Tang, ${ }^{\dagger, \nabla}$ Guohui Pan, ${ }^{\ddagger}$ Chin-Yuan Chang, ${ }^{\ddagger}$ and Ben Shen ${ }^{\star, \dagger, \downarrow, \cdot, \perp}$

${ }^{\dagger}$ Division of Pharmaceutical Sciences, School of Pharmacy, University of Wisconsin, Madison, Wisconsin 53705, United States, and ‡Department of Chemistry, "Department of Molecular Therapeutics, and $\perp^{N}$ atural Products Library Initiative at The Scripps Research Institute, The Scripps Research Institute, Jupiter, Florida 33458, United States.

\section{*E-mail: shenb@scripps.edu}

Present Addresses: ${ }^{\S}$ Xiangya International Academy of Translational Medicine, Central South University, Changsha, Hunan 410013, China; ${ }^{\nabla}$ State Key Laboratory of Bioorganic and Natural Product Chemistry, Shanghai Institute of Organic Chemistry, Chinese Academy of Sciences, 354 Fenglin Rd., Shanghai 200032, China.

Supporting Information

General experimental procedures S2

Plasmids for $\mathrm{Inml-KS1}$ and $\mathrm{Inml-KS2}$ site-directed mutagenesis and complementation Plasmids for Inml-ACP1 site-directed mutagenesis

Generation of the site-directed mutants and their complementation strains

Overproduction, purification and characterization of Lnml-ACP1 and its variants

Table S3 KS and ACP domains from selected NRPS-AT-less type I PKS machinery

Figure S1 Phylogenetic tree of KSs from selected NRPS-AT-less PKS machinery

Figure S2 Sequence analysis of selected KSs at the NRPS-AT-less PKS interfaces

Figure S3 Phylogenetic tree of ACPs from selected NRPS-AT-less PKS machinery 


\section{General experiment procedures}

The Streptomyces atroolivaceus S-140 wild-type strain ${ }^{\text {S1 }}$, E. coli S17-1, E. coli DH5a and E. coli BL21 (DE3) were used in this work. Plasmids pBS3005, pBS3006, pSET151, pSET152 and the ErmE $^{*}$ promoter were described previously. ${ }^{\mathrm{S2}, \mathrm{S} 3}$ Vectors pCR 2.1-TOPO, pET28a, pSP72, pBluescript II SK, pGEM-7Zf and pGEM-11Zf, as well as common chemicals and biochemicals were from commercial sources. Site-directed mutagenesis was performed using the QuikChange site-directed mutagenesis kit (Stratagene). Genetic manipulations in E. coli and S. atroolivaceus, conjugation between E. coli S17-1 and S. atroolivaceus, and selection of exconjugants, as well as Southern analysis were described previously. ${ }^{\mathrm{S} 3, \mathrm{~S} 4}$ Fermentation of the S. atroolivaceus S-140 wild-type and recombinant strains, and HPLC-MS analysis of leinamycin (LNM) production were carried out as previously reported. ${ }^{\text {S3 }}$

\section{Construction of pBS3118, pBS3119, pBS3120, and pBS3121 for Inml-KS1 and InmI-KS2 site-directed mutagenesis and complementation}

An 11.2-kb Kpnl fragment containing the Inml-KS1-ACP1-KS2 domains and flanking regions from cosmid pBS3006 was cloned into pSP72 to afford pSP72-InmI-KS1-ACP1-KS2. Then, a 2.3-kb Bglll-Xhol fragment encoding partial Lnml-NRPS module-2, a 2.2-kb BamHI-Xhol fragment containing partial $I n m I-K S 1-A C P 1$, and a 1.3-kb BamHI-Pstl fragment containing partial Inml-KS2 from pSP72-InmI-KS1-ACP1-KS2 were cloned into the corresponding sites of pBluescript II SK to afford pBS3112, pBS3113, and pBS3114, respectively.

pBS3116: A 2.1-kb Pstl-Sacl fragment from cosmid pBS3006 and a 0.3-kb Sacl-HindIII fragment from pBS3022 were first cloned into pSET151 to afford pBS3115. A 2.3-kb Xbal-Xhol fragment from pBS3112 and a 1.5-kb Sall-Pstl fragment containing the apramycin resistance gene aac(3)IV were cloned into the Xbal-Pstl sites of pBS3115 to yield pBS3116, in which the InmI-KS1-ACP1-KS2 region was replaced by aac(3)IV.

pBS3118: A 0.8-kb Xhol-Pstl fragment encoding partial Lnml-KS1 from pBS3113 was first cloned into pBluescript SK, the mutation C2090A was introduced by using the site-directed mutagenesis kit with PCR primers (5'-CC GTC GAC ACC TCC GCC TCG TCG TCG TTC G-3' and 5'-C GAA CGA CGA CGA GGC GGA GGT GTC GAC GG-3', the mutated codon is underlined), and the resultant plasmid was finally digested to afford a $0.5-\mathrm{kb}$ Xhol-ApaLI fragment. The aforementioned 0.5-kb Xhol-ApaLI fragment together with a 1.7-kb ApaLI-BamHI fragment from pBS3113 were cloned into the Xhol-BamHI sites of pBlueScript SK, and the resultant plasmid was digested by Xhol-BamHI to afford a 2.2-kb fragment. The aforementioned 2.2-kb fragment and a 2.3-kb Xbal-Xhol fragment from pBS3112 were then cloned into the Xbal-BamHI sites of pGEM-7Zf to afford pBS3117. pBS3117 was further digested with Xbal-BamHI to generate a 4.5-kb fragment. Finally, the aforementioned 4.5-kb fragment and a 1.2-kb BamHI-Pstl fragment from pBS3114 were cloned into the Pstl-Xbal sites of pBS3115 to afford pBS3118 (C2090A).

pBS3119 and pBS3120: In order to mutate Lnml-KS2 (C2824A), a 0.8-kb BamHI-Sacl fragment encoding partial Lnml-KS2 from pBS3114 was cloned into pSP72. The mutation C2824A was introduced by the site-directed mutagenesis kit with primers (5'-CC GTG GAC ACC ATG GCC TCG TCC TCG CTG AC-3' and 5'-GT CAG CGA GGA CGA GGC CAT GGT GTC CTC CAC GG-3', the mutated codon is underlined). The resultant plasmid was digested with BamHI-Sacl to give a 0.8-kb fragment, which was then cloned into pBS3114. The resultant plasmid was digested with BamHI-Pstl to afford a 1.2-kb fragment. This 1.2-kb fragment and the 4.5-kb Xbal-BamHI fragment from pBS3117 were cloned into pBS3115 to afford the final construct 
pBS3119 (C2824A). The Lnml-KS2 (H2959A) mutation was similarly introduced by the sitedirected mutagenesis kit with primers (5'-GC TAC GTG GAG GCG GCC GGC ACC GGC ACC C-3' and 5'-G GGT GCC GGT GCC GGC CGC CTC CAC GTA GC-3', the mutated codon is underlined). The resultant plasmid was digested with BamHI-Sacl to give a 0.8-kb fragment, which was then cloned into pBS3114. The resultant plasmid was digested with BamHI-Pstl to afford a 1.2-kb fragment. This 1.2-kb fragment and the 4.5-kb Xbal-BamHI fragment from pBS3117 were cloned into pBS3115 to afford the final construct pBS3120 (H2959A).

pBS3121: A 14.8-kb Bglll-Ndel DNA fragment containing intact $\mathrm{Inml}$ and its flanking regions from cosmid pBS3005 was cloned into the same sites of pUO9090, and the resultant plasmid was digested by EcoRI-Xbal to generate a 14.8-kb fragment. A 0.5-kb EcoRI-BamHI fragment containing ErmE* promoter was first cloned to Litmus 28, and the resultant plasmid was digested by EcoRI-Xbal to give the $0.5-\mathrm{kb} \mathrm{ErmE}^{*}$ fragment. This $0.5-\mathrm{kb}$ EcoRI-Xbal ErmE* fragment and the aforementioned 14.8-kb EcoRI-Xbal fragment were cloned into pSET152 to afford the final plasmid pBS3121, in which expression of $\mathrm{Inml}$ was under the control of ErmE* promoter.

Construction of pBS3130, pBS3132, pBS3135, pBS3136, pBS3137, and pBS3138 for Inm$A C P 1$ site-directed mutagenesis

The Inml-ACP1 domain was amplified from pBS3113 with primers ACP1-F (5'-CGGAATTCC ATA TGC GCG CGG ACC GCG CCC-3', EcoRI site underlined) and ACP1-R (5'-CGC AAGCTT GAC GGG CGG CGA CGG CT-3', HindIII site underlined), and then cloned into pSP72 to afford pBS3122.

pBS3123 and pBS3124: Mutation of the native Lnml-ACP1 $\left(\mathrm{S}_{2580} \mathrm{~S}_{2581}-\mathrm{S}_{2599} \mathrm{~S}_{2600}\right)$ to $\mathrm{Lnml}$ ACP1 $\left(\mathrm{A}_{2580} \mathrm{~A}_{2581}-\mathrm{S}_{2599} \mathrm{~S}_{2600}\right)$ on $\mathrm{pBS} 3122$ was achieved with primers ACP1M1-F (5'-CAC TAC GGC CTG GCC GCC CGC CTG GTC G-3', the mutated codon is underlined) and ACP1M1-R (5'-C GAC CAG GCG GGC GGC CAG GCC GTA GTG-3', the mutated codon is underlined) to afford pBS3123. Similarly, Lnml-ACP1 mutant variant $\left(D_{2580} S_{2581}-S_{2599} S_{2600}\right)$ was generated using pBS3122 as a template and primers ACP1M2-F (5'-CAC TAC GGC CTG GAC TCC CGC CTG GTC G-3', the mutated codon is underlined) and ACP1M2-R (5'-C GAC CAG GCG GGA GTC CAG GCC GTA GTG-3', the mutated codon is underlined), affording pBS3124.

pBS3125 and pBS3126: To construct the Lnml-ACP1 mutant variant $\left(A_{2580} A_{2581}-A_{2599} A_{2600}\right)$, overlap extension PCR with two pairs of primers, ACP1-F and ACP1M3-R (5'-GAA CAG GAC CGT GGC GGC CAC CCC TTG GAC-3', the mutated codon is underlined), ACP1-R and ACP1M3-F (5'-GTC CAA GGG GTG GCC GCC ACG GTC CTG TTC-3', the mutated codon is underlined) were carried out using pBS3123 as template. The two desired 0.2-kb PCR products were used as template for further PCR with primers ACP1-F and ACP1-R, and then the 0.4-kb PCR fragment was cloned to pSP72 to afford pBS3125. pBS3124 as an alternative template, similar procedure and same primers were used to generate Lnml-ACP1 mutant variant $\left(D_{2580} S_{2581}-A_{2599} A_{2600}\right)$, affording pBS3126.

pBS3127 and pBS3128: Similarly, to construct Lnml-ACP1 mutant variant $\left(\mathrm{A}_{2580} \mathrm{~A}_{2581^{-}}\right.$ $D_{2599} S_{2600}$ ), overlap extension PCR with two pairs of primers ACP1-F and ACP1M4-R (5'-GAA CAG GAC CGT GCT GTC CAC CCC TTG GAC-3', the mutated codon is underlined), ACP1-R and ACP1M4-F (5'-GTC CAA GGG GTG GAC AGC ACG GTC CTG TTC-3', the mutated codon is underlined) were carried out using pBS3123 as template. The two desired 0.2-kb PCR products were used as template for PCR with primers ACP1-F and ACP1-R, and then the 0.4-kb PCR fragment was cloned into pSP72 to afford pBS3127. With pBS3124 as an alternative 
template, similar procedure and same primers were used to generate Lnml-ACP1 mutant variant $\left(D_{2580} S_{2581}-D_{2599} S_{2600}\right)$, affording pBS3128.

pBS3130: A 1.0-kb Pstl-BamHI fragment from pBS3113 was first cloned into pSP72 to generate pBS3129. A 0.3-kb Pvull-Sall fragment from pBS3113, a 0.2-kb Pvull-Sall fragment from pBS3128, a 2.0-kb Xhol-Pvull fragment from pBS3113, and a 0.5-kb BspEl-BamHI fragment from pBS3129 were consecutively cloned into pSP72, resulting in a plasmid that was finally digested by Xhol-BamHI to give a 2.2-kb fragment. This 2.2-kb Xhol-BamHI fragment and a 2.3-kb Xbal-Xhol DNA fragment from pBS3112 were cloned into Xbal-BamHI sites of Litmus 28, and the resultant plasmid was further digested with Xbal-BamHI to generate a 4.4-kb fragment. This 4.4-kb Xbal-BamHI fragment and a 1.2-kb BamHI-Pstl DNA fragment from pBS3114 were finally cloned into the Xbal-Pstl sites of pBS3118 to afford pBS3130. pBS3130 was used to construct the Inml-ACP1 $\left(\mathrm{D}_{2580} \mathrm{~S}_{2581}-\mathrm{D}_{2599} \mathrm{~S}_{2600}\right)$ mutant strain SB3039 by homologous double crossover recombination in the $S$. atroolivaceus mutant strain SB3035.

pBS3132: A 0.3-kb Pvull-Sall fragment from pBS3113, a 0.2-kb Pvull-Sall fragment from pBS3127, a 2.0-kb Xhol-Pvull fragment from pBS3113, and a 0.5-kb BspEl-BamHI fragment from pBS3129 were consecutively cloned into pSP72 to afford pBS3131. Then, a 2.2-kb XholBamHI fragment from pBS3131 and a 2.3-kb Xbal-Xhol fragment from pBS3112 were cloned into Xbal-BamHI sites of Litmus 28 , and the resultant plasmid was further digested with XbalBamHI to generate a 4.4-kb fragment. This 4.4-kb Xbal-BamHI fragment and a 1.2-kb BamHIPstl fragment from pBS3114 were finally cloned into the Xbal-Pstl sites of pBS3118 to afford pBS3132. pBS3132 was used to construct the $I n m l-A C P 1\left(A_{2580} A_{2581}-D_{2599} S_{2600}\right)$ mutant strain SB3040 by homologous double crossover recombination in the $S$. atroolivaceus mutant strain SB3035.

pBS3135: A 2.1-kb Xhol-BamHI fragment from pBS3115 was first cloned into pSP72 to afford pBS3133. A 0.5-kb fragment was amplified from pBS3133 with primers KS1-Pstl (5'-CTG CAG ACC GGG CGC GC-3') and ACP1-M1-R. A 0.2-kb fragment was amplified from pBS3125 with primers ACP1M1-F and ACP1-RP2 (5'-GAT AAG CTT GAC GGG CGA CGG CT-3'). The two 0.5-kb and 0.2-kb fragments were used as templates for PCR with primers KS1-Pstl and ACP1RP2, and the resultant $0.7-\mathrm{kb}$ PCR products were cloned into the Pstl-HindIII sites of pSP72, affording a plasmid that was further digested by Pstl-BspEI to yield a $0.5-\mathrm{kb}$ fragment. This 0.5 $\mathrm{kb}$ fragment was then cloned into pBS3133, and the resultant plasmid was digested by XholAatll to afford a 1.2-kb fragment. This 1.2-kb fragment was then cloned into pBS3131 to afford pBS3134. A 2.2-kb Xhol-BamHI fragment from pBS3134 and a 2.3-kb Xbal-Xhol fragment from pBS3112 were cloned into Xbal-BamHI sites of pGEM-11Zf, yielding a plasmid that was further digested by Xbal-BamHI to afford a 4.4-kb fragment. Finally, this 4.4-kb fragment and a 1.2-kb BamHI-Pstl fragment from pBS3114 were cloned into the Xbal-Pstl sites of pBS3118 to afford pBS3135. pBS3135 was used to construct the Inml-ACP1 $\left(\mathrm{A}_{2580} \mathrm{~A}_{2581}-\mathrm{A}_{2599} \mathrm{~A}_{2600}\right)$ mutant strain SB3041 by homologous double crossover recombination in the $S$. atroolivaceus mutant strain SB3035.

pBS3136: A 2.1-kb Xhol-BamHl fragment from pBS3117 and a 2.0-kb Xhol-Aatll fragment from pBS3131 were cloned into pUO9090, and the resultant plasmid was digested by Xhol-BamHI to afford a 2.2-kb fragment. This 2.2-kb fragment and a 2.3-kb Xbal-Xhol fragment from pBS3112 were cloned into Xbal-BamHI sites of pGEM-11Zf, affording a plasmid that was further digested by $\mathrm{Xbal}-\mathrm{BamHI}$ to generate a $4.4-\mathrm{kb}$ fragment. Finally, this 4.4-kb fragment and a 1.2-kb BamHI-Pstl fragment from pBS3114 were cloned into the Xbal-Pstl sites of pBS3115 to afford pBS3136. pBS3136 was used to construct the InmI-ACP1 $\left(\mathrm{A}_{2580} \mathrm{~A}_{2581}-\mathrm{S}_{2599} \mathrm{~S}_{2600}\right)$ mutant strain SB3042 by homologous double crossover recombination in the $S$. atroolivaceus mutant strain 
SB3035.

pBS3137: A 2.1-kb Xhol-BamHI fragment from pBS3117 and a 0.5-kb Aatll-BamHI fragment from pBS3034 were cloned into pUO9090, and the resultant plasmid was further digested by Xhol-BamHI to generate a 2.2-kb fragment. This 2.2-kb fragment and a 2.3-kb Xbal-Xhol fragment from pBS3112 were cloned into Xbal-BamHI sites of pGEM-11Zf, affording a plasmid that was further digested by $\mathrm{Xbal}-\mathrm{BamHI}$ to generate a $4.4-\mathrm{kb}$ fragment. Finally, this $4.4-\mathrm{kb}$ fragment and a 1.2-kb BamHI-Pstl fragment from pBS3114 were cloned into the Xbal-Pstl sites of pBS3115 to afford pBS3137. pBS3137 was used to construct the Inml-ACP1 $\left(\mathrm{S}_{2580} \mathrm{~S}_{2581^{-}}\right.$ $\mathrm{A}_{2599} \mathrm{~A}_{2600}$ ) mutant strain SB3043 by homologous double crossover recombination in the $S$. atroolivaceus mutant strain SB3035.

pBS3138: A 2.0-kb Xhol-Aatll fragment from pBS3126 was first cloned into pBS3134, and the resultant plasmid was digested by Xhol-BamHI to afford a 2.2-kb fragment. Then this 2.2-kb fragment and a 2.3-kb Xbal-Xhol fragment from pBS3112 were cloned into Xbal-BamHI sites of pGEM-11Zf, affording a plasmid that was further digested by Xbal-BamHI to generate a 4.4-kb fragment. Finally, this 4.4-kb fragment and a 1.2-kb BamHI-Pstl fragment from pBS3114 were cloned into the Xbal-Pstl sites of pBS3115 to afford pBS3138. pBS3138 was used to construct the Inml-ACP1 $\left(\mathrm{D}_{2580} \mathrm{~S}_{2581}-\mathrm{A}_{2599} \mathrm{~A}_{2600}\right)$ mutant strain SB4144 by homologous double crossover recombination in the $S$. atroolivaceus mutant strain SB3035.

Generation of site-directed S. atroovaceus mutants SB3036, SB3037, SB3038, SB3039, SB3040, SB3041, SB3042, SB3043, SB3044 and their complementation strains SB3045, SB3046, and SB3047

pBS3116 was introduced into $S$. atroolivaceus S-140 wild-type by conjugation. After several rounds of passaging, the exconjugants on solid TSB media, i.e., the double-crossover mutants that were apramycin resistant and thiostrepton sensitive, were obtained and named $S$. atroolivaceus SB3035, whose genotype, with the InmI-KS1-ACP1-KS2 region replaced by the aac(3)IV cassette, was confirmed by Southern analysis. Plasmids pBS3118, pBS3119, pBS3120, pBS3130, pBS3132, pBS3135, pBS3136, pBS3137, and pBS3138 with respective point mutations for $I n m l-K S 1,-K S 2$, or $-A C P 1$ were introduced into the SB3035 strain by conjugation. After several rounds of passaging, the exconjugants on solid TSB media, i.e., the desired double-crossover mutants that were apramycin sensitive and thiostrepton sensitive, were obtained, respectively, and their respective genotypes were confirmed by southern analysis. These $S$. atroolivaceus mutants were named SB3036 (C2090A), SB3037 (H2959A), SB3038 (C2824A), SB3039 ( $\left(D_{2580} S_{2581}-D_{2599} S_{2600}\right)$, SB3040 $\left(A_{2580} A_{2581}-D_{2599} S_{2600}\right)$, SB3041 $\left(A_{2580} A_{2581}-A_{2599} A_{2600}\right)$, SB3042 $\left(A_{2580} A_{2581}-S_{2599} S_{2600}\right), \quad S B 3043 \quad\left(S_{2580} S_{2581}-A_{2599} A_{2600}\right)$, and SB3044 $\left(D_{2580} S_{2581}-A_{2599} A_{2600}\right)$, respectively.

Introduction of complementation plasmid pBS3121 to InmI-KS1 mutant SB3036 (C2090A) or Inml-KS2 mutants SB3037 (H2959A) and SB3038 (C2824A) was carried out by conjugation, affording the three complementation mutant strains SB3045 (C2090A), SB3046 (H2959A), and SB3047 (C2824A), respectively.

Construction of pBS3139, pBS3140, pBS3141, pBS3142, and pBS3143 for overproduction, purification and characterization of Lnml-ACP1 and its variants

Four fragments of the wild-type Inml-ACP1 $\left(\mathrm{S}_{2580} \mathrm{~S}_{2581}-\mathrm{S}_{2599} \mathrm{~S}_{2600}\right)$ and its mutant variants $\left(\mathrm{D}_{2580} \mathrm{~S}_{2581}-\mathrm{A}_{2599} \mathrm{~A}_{2600}\right),\left(\mathrm{S}_{2580} \mathrm{~S}_{2581}-\mathrm{A}_{2599} \mathrm{~A}_{2600}\right)$, and $\left(\mathrm{A}_{2580} \mathrm{~A}_{2581}-\mathrm{D}_{2599} \mathrm{~S}_{2600}\right)$ were amplified by PCR from the genomic DNAs of $S$. atroolivaceus S-140 $\left(\mathrm{S}_{2580} \mathrm{~S}_{2581}-\mathrm{S}_{2599} \mathrm{~S}_{2600}\right)$, SB3044 $\left(\mathrm{D}_{2580} \mathrm{~S}_{2581^{-}}\right.$ 
$\left.A_{2599} A_{2600}\right)$, SB3043 $\left(S_{2580} S_{2581}-A_{2599} A_{2600}\right)$, and SB3040 ( $\left.A_{2580} A_{2581}-D_{2599} S_{2600}\right)$, respectively, using primers ACP1-mutation-F (5'-CAT GAATTC CATATG GCC GCG CCC GCG GCC-3', EcoRI and Ndel sites underlined) and ACP1-mutation-R (5'-CAT AAGCTT GGG ACC GTC GGC CGC GG-3', HindlII site underlined). The resultant four PCR products, termed as ACP1-I to ACP1-IV, were digested with Ndel-HindIII and cloned into pET28a to generate the expression plasmids pBS3139 $\left(\mathrm{S}_{2580} \mathrm{~S}_{2581}-\mathrm{S}_{2599} \mathrm{~S}_{2600}\right)$, pBS3140 $\left(\mathrm{D}_{2580} \mathrm{~S}_{2581}-\mathrm{A}_{2599} \mathrm{~A}_{2600}\right)$, pBS3141 $\left(\mathrm{S}_{2580} \mathrm{~S}_{2581^{-}}\right.$ $\left.A_{2599} A_{2600}\right)$, and pBS3142 $\left(A_{2580} A_{2581}-D_{2599} S_{2600}\right)$, respectively. For construction of the expression plasmid pBS3143 $\left(D_{2580} S_{2581}-S_{2599} S_{2600}\right)$, fragments ACP1-I and ACP1-II were similarly digested with Ndel-HindIII, and the resultant fragments were cloned into pCR 2.1-TOPO to afford pCR 2.1-TOPO-ACP1-I and pCR 2.1-TOPO-ACP1-II, respectively. A 0.4-kb Ndel-Aatll fragment from pCR 2.1-TOPO-ACP1-II was cloned into the Ndel-Aatll sites of pCR 2.1-TOPO-ACP1-I, and the resultant plasmid was further digested with Ndel-HindIII to generate a 0.4-kb fragment. Finally this 0.4-kb Ndel-HindIII fragment was cloned into pET28a to afford pBS3143 $\left(\mathrm{D}_{2580} \mathrm{~S}_{2581^{-}}\right.$ $\left.\mathrm{S}_{2599} \mathrm{~S}_{2600}\right)$.

The expression plasmids of the Inml-ACP1 wild-type and mutant variants were introduced into E. coli BL21 (DE3), and the protein production was induced by $0.1 \mathrm{mM}$ Isopropyl- $\beta$-Dthiogalactopyranoside (IPTG) at $30{ }^{\circ} \mathrm{C}$. Protein was purified by Ni-NTA affinity chromatography, and further dialyzed (1 mM EDTA, $1 \mathrm{mM}$ DTT, 10\% glycerol, $10 \mathrm{mM}$ Tris $\bullet \mathrm{HCl}, \mathrm{pH}$ 8.0) and stored at $-80{ }^{\circ} \mathrm{C}$. The isolated yields for Lnml-ACP1 and its mutant variants were estimated to be $10 \mathrm{mg} / \mathrm{L}$. Sfp and TcmM ACP was produced and purified as described previously. ${ }^{\mathrm{S}}$

In vitro phosphopantetheinylation reaction consisted of $50 \mu \mathrm{M}$ TcmM ACP, or $60 \mu \mathrm{M}$ apo-LnmlACP1 or mutant variants, $1 \mu \mathrm{M}$ Sfp, $250 \mu \mathrm{M}$ CoA, $5 \mathrm{mM}$ dithiothreitol, $12.5 \mathrm{mM} \mathrm{MgCl}, 75 \mathrm{mM}$ Tris $\cdot \mathrm{HCl}, \mathrm{pH}$ 8.0, and the final reaction volume was $50 \mu \mathrm{L}$. The reaction was initiated by the addition of CoA, further incubated at $37{ }^{\circ} \mathrm{C}$ for $15 \mathrm{~min}(\mathrm{TcmM}$ ) or $120 \mathrm{~min}$ (for Lnml-ACP1 and its mutant variants), and terminated by addition of EDTA to a final concentration of $50 \mathrm{mM}$ and then immediately frozen at $-80{ }^{\circ} \mathrm{C}$. The samples were analyzed on an analytic Jupiter $\mathrm{C}-18$ column $(5 \mu \mathrm{m}, 300 \AA, 250 \times 4.6 \mathrm{~mm}$, Phenomenex, Belmont, CA). The column was equilibrated with $100 \%$ solvent $A\left(\mathrm{H}_{2} \mathrm{O}\right.$ with $0.1 \%$ trifluoroacetic acid) and developed with a 20 min linear gradient from $0 \% \mathrm{~B}$ (acetonitrile with $0.1 \%$ trifluoroacetic acid) to $100 \% \mathrm{~B}$ at a flow rate of 1 $\mathrm{mL} / \mathrm{min}$. The peaks corresponding to ACPs were collected, lyophilized, and analyzed by ESIMS as previously described. ${ }^{\text {S6 }}$

\section{References}

S1. Hara, M.; Asano, K.; Kawamoto, I.; Takiguchi, T.; Katsumata, S.; Takahashi, K. I.; Nakano, H. J. Antibiotics 1989,42, 1768-1774.

S2. Cheng, Y. Q.; Tang, G. L.; Shen, B. J. Bacteriol. 2002, 184, 7013-7024.

S3. Tang, G. L.; Cheng, Y. Q.; Shen, B. Chem. Biol. 2004, 11, 33-45.

S4. Tang, G. L.; Cheng, Y. Q.; Shen, B. J. Nat. Prod. 2006, 69, 387-393.

S5. Sánchez, C.; Du, L.; Edwards, D.J.; Toney, M.D.; Shen, B. Chem. Biol. 2001, 8, 725-738.

S6. Huang, Y.; Wendt-Pienkowski, E.; Shen, B. J. Biol. Chem. 2006, 281, 29660-29668. 
Table S1. Plasmids used in this study.

\begin{tabular}{|c|c|c|}
\hline Plasmids & Description & Reference \\
\hline pBS3005 & Cosmid containing partial Inm gene cluster & S2 \\
\hline pBS3006 & Cosmid containing partial Inm gene cluster & S2 \\
\hline pBS3112 & pBluescript II SK derivative with a 2.3-kb fragment encoding partial Lnml-NRPS module-2 & This work \\
\hline pBS3113 & pBluescript II SK derivative with a 2.2-kb fragment containing partial InmI-KS1-ACP1 & This work \\
\hline pBS3114 & pBluescript II SK derivative with a 1.3-kb fragment containing partial $/ \mathrm{nm} /-K S 2$ & This work \\
\hline pBS3115 & pSET151 derivative plasmid with a 2.4-kb downstream DNA region of $\mathrm{Inml-KS2}$ & This work \\
\hline pBS3116 & pSET151 derivative plasmid for inactivation of $I n m I ~ K S 1-A C P 1-K S 2$ in vivo & This work \\
\hline pBS3117 & $\begin{array}{l}\text { pGEM-7Zf derivative with } 4.5-\mathrm{kb} \text { region containing } I n m I-K S 1-A C P 1 \text { and its flanking } \\
\text { regions }\end{array}$ & This work \\
\hline pBS3118 & pSET151 derivative plasmid for introducing the point mutation C2090A in $\mathrm{Inml-KS1} \mathrm{in} \mathrm{vivo}$ & This work \\
\hline pBS3119 & pSET151 derivative plasmid for introducing the point mutation C2824A in $/ \mathrm{nm} /-K S 2$ in vivo & This work \\
\hline pBS3120 & pSET151 derivative plasmid for introducing the point mutation $\mathrm{H} 2959 \mathrm{~A}$ in $\mathrm{Inm} / \mathrm{KS} 2$ in vivo & This work \\
\hline pBS3121 & pSET152 derivative plasmid with ErmE $^{*}$ promoter for over-expression of $I n m I$ & This work \\
\hline pBS3122 & pSP72 derivative plasmid containing the wild-type $/ \mathrm{nm} / \mathrm{ACP} 1$ & This work \\
\hline pBS3123 & pSP72 derivative plasmid containing the mutant variant $I n m l-A C P 1\left(\mathrm{~A}_{2580} \mathrm{~A}_{2581}-\mathrm{S}_{2599} \mathrm{~S}_{2600}\right)$ & This work \\
\hline pBS3124 & pSP72 derivative plasmid containing the mutant variant $\operatorname{lnml}-A C P 1\left(\mathrm{D}_{2580} \mathrm{~S}_{2581}-\mathrm{S}_{2599} \mathrm{~S}_{2600}\right)$ & This work \\
\hline pBS3125 & pSP72 derivative plasmid containing the mutant variant $I n m l-A C P 1\left(\mathrm{~A}_{2580} \mathrm{~A}_{2581}-\mathrm{A}_{2599} \mathrm{~A}_{2600}\right)$ & This work \\
\hline pBS3126 & pSP72 derivative plasmid containing the mutant variant $I n m l-A C P 1\left(D_{2580} S_{2581}-A_{2599} A_{2600}\right)$ & This work \\
\hline pBS3127 & pSP72 derivative plasmid containing the mutant variant Inml-ACP1 $\left(\mathrm{A}_{2580} \mathrm{~A}_{2581}-\mathrm{D}_{2599} \mathrm{~S}_{2600}\right.$ & This work \\
\hline pBS3128 & pSP72 derivative plasmid containing the variant $I n m l-A C P 1\left(\mathrm{D}_{2580} \mathrm{~S}_{2581}-\mathrm{D}_{2599} \mathrm{~S}_{2600}\right)$ & This work \\
\hline pBS3129 & pSP72 derivative plasmid used for construction of pBS3130 and pBS3132 & This work \\
\hline pBS3130 & $\begin{array}{l}\text { pSET151 derivative plasmid for generating the } I n m l-A C P 1 \text { variant mutation }\left(D_{2580} S_{2581^{-}}\right. \\
\left.D_{2599} S_{2600}\right) \text { in vivo }\end{array}$ & This work \\
\hline pBS3131 & pBS3129 derivative plasmid used for construction of pBS3132 and others & This work \\
\hline pBS3132 & $\begin{array}{l}\text { pSET151 derivative plasmid for generating the } \operatorname{lnml}-A C P 1 \text { variant mutation }\left(A_{2580} A_{2581^{-}}\right. \\
\left.D_{2599} S_{2600}\right) \text { in vivo }\end{array}$ & This work \\
\hline pBS3133 & pSP72p derivative plasmid used for construction of pBS3135 & This work \\
\hline pBS3134 & pBS3131 derivative plasmid used for construction of pBS3135 & This work \\
\hline pBS3135 & $\begin{array}{l}\text { pSET151 derivative plasmid for generating the } \operatorname{lnml}-A C P 1 \text { variant mutation }\left(\mathrm{A}_{2580} \mathrm{~A}_{2581^{-}}\right. \\
\left.\mathrm{A}_{2599} \mathrm{~A}_{2600}\right) \text { in vivo }\end{array}$ & This work \\
\hline pBS3136 & $\begin{array}{l}\text { pSET151 derivative plasmid for generating the } \operatorname{lnml}-A C P 1 \text { variant mutation }\left(\mathrm{A}_{2580} \mathrm{~A}_{2581^{-}}\right. \\
\left.\mathrm{S}_{2599} \mathrm{~S}_{2600}\right) \text { in vivo }\end{array}$ & This work \\
\hline pBS3137 & $\begin{array}{l}\text { pSET151 derivative plasmid for generating the } \operatorname{lnml}-A C P 1 \text { variant mutation }\left(\mathrm{S}_{2580} \mathrm{~S}_{2581^{-}}\right. \\
\left.\mathrm{A}_{2599} \mathrm{~A}_{2600}\right) \text { in vivo }\end{array}$ & This work \\
\hline pBS3138 & $\begin{array}{l}\text { pSET151 derivative plasmid for generating the } I n m l-A C P 1 \text { variant mutation }\left(D_{2580} S_{2581^{-}}\right. \\
\left.A_{2599} A_{2600}\right) \text { in vivo }\end{array}$ & This work \\
\hline pBS3139 & $\begin{array}{l}\text { pET28a derivative plasmid for overproduction of the wild-type Lnml-ACP1 }\left(\mathrm{S}_{2580} \mathrm{~S}_{2581^{-}}\right. \\
\left.\mathrm{S}_{2599} \mathrm{~S}_{2600}\right)\end{array}$ & This work \\
\hline pBS3140 & $\begin{array}{l}\text { pET28a derivative plasmid for overproduction of the mutant Lnml-ACP1 }\left(D_{2580} S_{2581^{-}}\right. \\
\left.A_{2599} A_{2600}\right)\end{array}$ & This work \\
\hline pBS3141 & $\begin{array}{l}\text { pET28a derivative plasmid for overproduction of the mutant Lnml-ACP1 }\left(\mathrm{S}_{2580} \mathrm{~S}_{2581^{-}}\right. \\
\left.\mathrm{A}_{2599} \mathrm{~A}_{2600}\right)\end{array}$ & This work \\
\hline pBS3142 & $\begin{array}{l}\text { pET28a derivative plasmid for overproduction of the mutant Lnml-ACP1 }\left(A_{2580} A_{2581-}\right. \\
\left.D_{2599} S_{2600}\right)\end{array}$ & This work \\
\hline pBS3143 & $\begin{array}{l}\text { pET28a derivative plasmid for overproduction of the mutant Lnml-ACP1 }\left(\mathrm{D}_{2580} \mathrm{~S}_{2581^{-}}\right. \\
\left.\mathrm{S}_{2599} \mathrm{~S}_{2600}\right)\end{array}$ & This work \\
\hline
\end{tabular}


Table S2. Bacterial strains used in this study.

\begin{tabular}{|c|c|c|}
\hline Strains & Description & References \\
\hline $\begin{array}{l}\text { S. atroolivaceus } \\
\text { S-140 }\end{array}$ & LNM producing strain, wild-type & S1 \\
\hline SB3035 & S. atroolivaceus mutant strain, $I \mathrm{~nm} /$ inactivated by insertion of the aac(3)IV gene & This work \\
\hline SB3036 & S. atroolivaceus mutant strain with point mutation in InmI-KS1 (C2090A) & This work \\
\hline SB3037 & S. atroolivaceus mutant strain with point mutation in InmI-KS2 (H2959A) & This work \\
\hline SB3038 & S. atroolivaceus mutant strain with point mutation in InmI-KS2 (C2824A) & This work \\
\hline SB3039 & S. atroolivaceus mutant strain with mutations in $\operatorname{lnml}-A C P 1\left(\mathrm{D}_{2580} \mathrm{~S}_{2581}-\mathrm{D}_{2599} \mathrm{~S}_{2600}\right)$ & This work \\
\hline SB3040 & S. atroolivaceus mutant strain with mutations in Inml-ACP1 $\left(\mathrm{A}_{2580} \mathrm{~A}_{2581}-\mathrm{D}_{2599} \mathrm{~S}_{2600}\right)$ & This work \\
\hline SB3041 & S. atroolivaceus mutant strain with mutations in Inml-ACP1 $\left(\mathrm{A}_{2580} \mathrm{~A}_{2581}-\mathrm{A}_{2599} \mathrm{~A}_{2600}\right)$ & This work \\
\hline SB3042 & S. atroolivaceus mutant strain with mutations in $\operatorname{lnml}-A C P 1\left(\mathrm{~A}_{2580} \mathrm{~A}_{2581}-\mathrm{S}_{2599} \mathrm{~S}_{2600}\right)$ & This work \\
\hline SB3043 & S. atroolivaceus mutant strain with mutations in $\operatorname{lnml}-A C P 1\left(\mathrm{~S}_{2580} \mathrm{~S}_{2581}-\mathrm{A}_{2599} \mathrm{~A}_{2600}\right)$ & This work \\
\hline SB3044 & S. atroolivaceus mutant strain with mutations in $\operatorname{lnml}-A C P 1\left(\mathrm{D}_{2580} \mathrm{~S}_{2581}-\mathrm{A}_{2599} \mathrm{~A}_{2600}\right)$ & This work \\
\hline SB3045 & SB3036 complemented with pBS3121 & This work \\
\hline SB3046 & SB3037 complemented with pBS3121 & This work \\
\hline SB3047 & SB3038 complemented with pBS3121 & This work \\
\hline
\end{tabular}


Table S3 KS and ACP domains from selected NRPS-AT-less type I PKS machinery ${ }^{a}$

\section{Canonical NRPS-PKS interface}

NRPS
module $\begin{gathered}\text { Type IPKS } \\ \text { module }\end{gathered}$

NRPS-Type I PKS

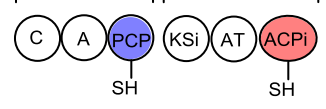

$$
\begin{array}{cc}
\text { AT-less } \\
\text { NRPS } \\
\text { type IPKS }
\end{array}
$$

module module

NRPS-AT-less Type I PKS

$$
\text { (C) A) }
$$

NRPS-PKS interface featuring

"KS-ACP-KS" domain architecture

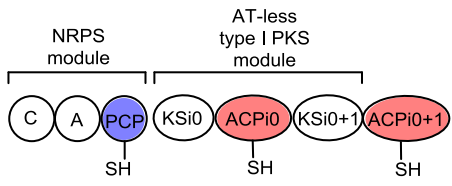

"KS-ACP-KS" domain architecture at the LnmI NRPS-AT-less type I PKS interface

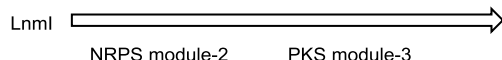

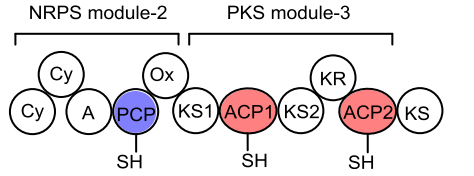

$\mathrm{KSi} 0=\mathrm{KS} 1$

$\mathrm{ACPiO}=\mathrm{ACP} 1$

$\mathrm{KSIO}+1=\mathrm{KS} 2$
$\mathrm{ACP} O+1=\mathrm{ACP} 2$ functions at the interface of NRPS-PKS; KSi0: proposed non-elongating KSs at the interface of NRPS-PKS; KSi0+1: the KS located downstream of a KSiO; KS0: non-elongating KSs except the ones at the interface of NRPS-PKS.

\begin{tabular}{|c|c|c|c|c|c|c|c|c|c|c|c|c|c|c|c|c|}
\hline accession & cluster & name & $\mathrm{KS}^{\mathrm{b}}$ & KSi & KSiO & $\mathrm{KSi0}+1$ & KSO & KS start & KS end & ACP & $\mathrm{ACPi}$ & ACPi0 & $\mathrm{ACPi} 0+1$ & ACP0 & ACP start & ACP end \\
\hline CAB13589 & Bacillaene & PksJ & & KS1 & & & & 1758 & 2197 & & ACP1 & & & & 3112 & 3186 \\
\hline CAB13589 & Bacillaene & PksJ & KS2 & & & & & 3337 & 3790 & ACP2 & & & & & 4462 & 4534 \\
\hline CAB13589 & Bacillaene & PksJ & & & & & KS3 & 4586 & 5003 & & & & & & & \\
\hline CAB13602 & Bacillaene & PksL & & & & & & & & & & & & ACP3 & 320 & 393 \\
\hline CAB13602 & Bacillaene & PksL & KS4 & & & & & 431 & 882 & ACP4 & & & & & 1804 & 1869 \\
\hline CAB13602 & Bacillaene & PksL & KS5 & & & & & 1924 & 2376 & ACP5 & & & & & 2741 & 2815 \\
\hline$\overline{C A B 13602}$ & Bacillaene & PksL & KS6 & & & & & 2871 & 3305 & ACP6 & & & & & 3966 & 4036 \\
\hline CAB13602 & Bacillaene & $\mathrm{PksL}$ & & & & & KS7 & 4080 & 4496 & & & & & & & \\
\hline CAB13603 & Bacillaene & PksM & & & & & & & & & & & & ACP7 & 298 & 365 \\
\hline CAB13603 & Bacillaene & PksM & KS8 & & & & & 391 & 842 & ACP8 & & & & & 2187 & 2256 \\
\hline CAB13603 & Bacillaene & PksM & KS9 & & & & & 2317 & 2745 & ACP9 & & & & & 3411 & 3478 \\
\hline CAB13603 & Bacillaene & PksM & KS10 & & & & & 3527 & 3955 & ACP10 & & & & & 4132 & 4209 \\
\hline CAB13604 & Bacillaene & PksN & & KS11 & & & & 1087 & 1526 & & ACP11 & & & & 2445 & 2524 \\
\hline$\overline{\mathrm{CAB} 13604}$ & Bacillaene & PksN & KS12 & & & & & 2574 & 3023 & ACP12 & & & & & 3943 & 4025 \\
\hline$\overline{\mathrm{CAB} 13604}$ & Bacillaene & PksN & KS13 & & & & & 4074 & 4522 & & & & & & & \\
\hline$\overline{\mathrm{CAB} 13606}$ & Bacillaene & PksR & & & & & & & & ACP13 & & & & & 375 & 449 \\
\hline CAB13606 & Bacillaene & PksR & & & & & KS14 & 525 & 970 & & & & & ACP14 & 1407 & 1486 \\
\hline CAB13606 & Bacillaene & PksR & KS15 & & & & & 1526 & 1957 & ACP15 & & & & & 2143 & 2204 \\
\hline ADD82939 & Batumin & Bat1 & KS1 & & & & & 561 & 1001 & ACP1 & & & & & 2102 & 2181 \\
\hline$\overline{\text { ADD82940 }}$ & Batumin & Bat2 & & KS2 & & & & 1136 & 1576 & & ACP2 & & & & 2268 & 2352 \\
\hline ADD82940 & Batumin & Bat2 & KS3 & & & & & 2406 & 2845 & ACP3 & & & & & 3500 & 3566 \\
\hline$\overline{\text { ADD82940 }}$ & Batumin & Bat2 & KS4 & & & & & 3619 & 4070 & ACP4 & & & & & 4308 & 4389 \\
\hline ADD82940 & Batumin & Bat2 & KS5 & & & & & 4732 & 5168 & ACP5 & & & & & 6098 & 6176 \\
\hline$\overline{\text { ADD82940 }}$ & Batumin & Bat2 & KS6 & & & & & 6240 & 6692 & ACP6 & & & & & 7375 & 7462 \\
\hline ADD82940 & Batumin & Bat2 & & & & & KS7 & 7515 & 7932 & & & & & & & \\
\hline ADD82941 & Batumin & Bat3 & & & & & & & & & & & & ACP7 & 305 & 378 \\
\hline$\overline{\text { ADD82941 }}$ & Batumin & Bat3 & KS8 & & & & & 426 & 875 & ACP8 & & & & & 1815 & 1895 \\
\hline ADD82941 & Batumin & Bat3 & KS9 & & & & & 1957 & 2409 & ACP9 & & & & & 2868 & 2939 \\
\hline
\end{tabular}

${ }^{b} \mathrm{KS}$ : all the other KSs except the above-mentioning KSs. ACP, ACPi, ACPi0, ACPi0+1 and ACP0 corresponds to the ACPs from the same module with KS, KSi, KSi0, KSi0+1, respectively. 


\begin{tabular}{|c|c|c|c|c|c|c|c|c|c|c|c|c|c|c|c|c|}
\hline ADD82941 & Batumin & Bat3 & KS10 & & & & & 3270 & 3703 & ACP10 & & & & & 3972 & 4045 \\
\hline ADD82941 & Batumin & Bat3 & KS11 & & & & & 4113 & 4549 & ACP11 & & & & & 4796 & 4867 \\
\hline BAP05589 & Calyculin A & CalA & & & KS1 & & & 1481 & 1899 & & & ACP1 & & & 2085 & 2174 \\
\hline BAP05589 & Calyculin A & CalA & & & & KS2 & & 2240 & 2670 & & & & ACP2 & & 3704 & 3776 \\
\hline BAP05590 & Calyculin A & CalB & & & & & KS3 & 77 & 498 & & & & & ACP3 & 660 & 747 \\
\hline BAP05590 & Calyculin A & CalB & & KS4 & & & & 1850 & 2271 & & ACP4 & & & & 3644 & 3710 \\
\hline BAP05590 & Calyculin A & CalB & & & & & KS5 & 4227 & 4635 & & & & & ACP5 & 4832 & 4912 \\
\hline BAP05590 & Calyculin A & CalB & & & & & KS6 & 4970 & 5394 & & & & & ACP6 & 5456 & 5522 \\
\hline BAP05591 & Calyculin A & CalC & & & KS7 & & & 1116 & 1531 & & & ACP7 & & & 1770 & 1775 \\
\hline BAP05591 & Calyculin A & CalC & & & & KS8 & & 1815 & 2232 & & & & ACP8 & & 3151 & 3215 \\
\hline BAP05591 & Calyculin A & CalC & KS9 & & & & & 3276 & 3695 & ACP9 & & & & & 4542 & 4620 \\
\hline BAP05593 & Calyculin A & CalE & & & & & KS10 & 64 & 482 & & & & & ACP10 & 931 & 1014 \\
\hline BAP05593 & Calyculin A & CalE & KS11 & & & & & 1057 & 1485 & ACP11 & & & & & 1678 & 1761 \\
\hline BAP05593 & Calyculin A & CalE & KS12 & & & & & 1812 & 2245 & ACP12 & & & & & 3320 & 3393 \\
\hline BAP05593 & Calyculin A & CalE & KS13 & & & & & 3438 & 3837 & ACP13 & & & & & 4459 & 4535 \\
\hline BAP05593 & Calyculin A & CalE & & & & & KS14 & 4579 & 5004 & & & & & ACP14 & 5197 & 5269 \\
\hline BAP05593 & Calyculin A & CalE & KS15 & & & & & 5312 & 5723 & ACP15 & & & & & 6430 & 6516 \\
\hline BAP05593 & Calyculin A & CalE & KS16 & & & & & 6687 & 7100 & ACP16 & & & & & 7850 & 7924 \\
\hline BAP05594 & Calyculin A & CalF & & & & & KS17 & 74 & 499 & & & & & ACP17 & 673 & 754 \\
\hline$\overline{\text { BAP05594 }}$ & Calyculin A & CalF & KS18 & & & & & 783 & 1197 & ACP18 & & & & & 1912 & 1995 \\
\hline BAP05594 & Calyculin A & CalF & & & & & KS19 & 2053 & 2468 & & & & & ACP19 & 2981 & 3061 \\
\hline BAP05594 & Calyculin A & CalF & KS20 & & & & & 3123 & 3534 & ACP20 & & & & & 4623 & 4693 \\
\hline BAP05594 & Calyculin A & CalF & KS21 & & & & & 4754 & 5151 & & & & & & & \\
\hline BAP05595 & Calyculin A & CalG & & & & & & & & ACP21 & & & & & 404 & 484 \\
\hline BAP05595 & Calyculin A & CalG & KS22 & & & & & 553 & 962 & ACP22 & & & & & 2423 & 2500 \\
\hline BAP05595 & Calyculin A & CalG & KS23 & & & & & 2567 & 2986 & ACP23 & & & & & 3280 & 3335 \\
\hline BAP05595 & Calyculin A & CalG & KS24 & & & & & 3589 & 4007 & ACP24 & & & & & 5044 & 5121 \\
\hline BAP05595 & Calyculin A & CalG & KS25 & & & & & 5163 & 5592 & & & & & & & \\
\hline BAP05596 & Calyculin A & CalH & & & & & & & & ACP25 & & & & & 578 & 656 \\
\hline BAP05596 & Calyculin A & CalH & KS26 & & & & & 836 & 1268 & ACP26 & & & & & 1458 & 1548 \\
\hline BAP05596 & Calyculin A & CalH & & KS27 & & & & 2708 & 3128 & & ACP27 & & & & 4056 & 4119 \\
\hline BAP05596 & Calyculin A & CalH & & & & & KS28 & 4187 & 4613 & & & & & & & \\
\hline$\overline{\text { BAP05597 }}$ & Calyculin A & Call & & & & & & & & & & & & ACP28 & 323 & 406 \\
\hline BAP05597 & Calyculin A & Call & KS29 & & & & & 479 & 895 & ACP29 & & & & & 1214 & 1292 \\
\hline CAN94300 & Chivosazol & ChiB & KS1 & & & & & 109 & 549 & ACP1 & & & & & 1860 & 1941 \\
\hline CAN94300 & Chivosazol & ChiB & KS2 & & & & & 2001 & 2446 & ACP2 & & & & & 3676 & 3758 \\
\hline$\overline{\text { CAN94300 }}$ & Chivosazol & ChiB & KS3 & & & & & 3838 & 4276 & ACP3 & & & & & 5690 & 5771 \\
\hline CAN94299 & Chivosazol & ChiC & KS4 & & & & & 66 & 502 & ACP4 & & & & & 1232 & 1299 \\
\hline CAN94299 & Chivosazol & ChiC & KS5 & & & & & 1459 & 1892 & ACP5 & & & & & 2279 & 2343 \\
\hline CAN94299 & Chivosazol & ChiC & KS6 & & & & & 3015 & 3455 & ACP6 & & & & & 4450 & 4524 \\
\hline$\overline{\text { CAN94299 }}$ & Chivosazol & ChiC & KS7 & & & & & 4610 & 5055 & ACP7 & & & & & 5835 & 5907 \\
\hline CAN94299 & Chivosazol & ChiC & KS8 & & & & & 5953 & 6401 & ACP8 & & & & & 7509 & 7586 \\
\hline CAN94299 & Chivosazol & ChiC & & & & & KS9 & 7631 & 8075 & & & & & ACP9 & 8282 & 8346 \\
\hline CAN94298 & Chivosazol & ChiD & & & KS10 & & & 2029 & 2466 & & & ACP10 & & & 2677 & 2755 \\
\hline CAN94298 & Chivosazol & ChiD & & & & KS11 & & 2798 & 3246 & & & & ACP11 & & 3993 & 4066 \\
\hline$\overline{\text { CAN94298 }}$ & Chivosazol & ChiD & KS12 & & & & & 4149 & 4581 & & & & & & & \\
\hline CAN94297 & Chivosazol & ChiE & & & & & & & & ACP12 & & & & & 358 & 436 \\
\hline CAN94297 & Chivosazol & ChiE & KS13 & & & & & 985 & 1430 & ACP13 & & & & & 2662 & 2747 \\
\hline CAN94297 & Chivosazol & ChiE & KS14 & & & & & 2787 & 3216 & ACP14 & & & & & 4631 & 4706 \\
\hline$\overline{\text { CAN94296 }}$ & Chivosazol & ChiF & KS15 & & & & & 27 & 472 & ACP15 & & & & & 1178 & 1256 \\
\hline CAN94296 & Chivosazol & ChiF & KS16 & & & & & 1305 & 1737 & ACP16 & & & & & 2110 & 2183 \\
\hline
\end{tabular}




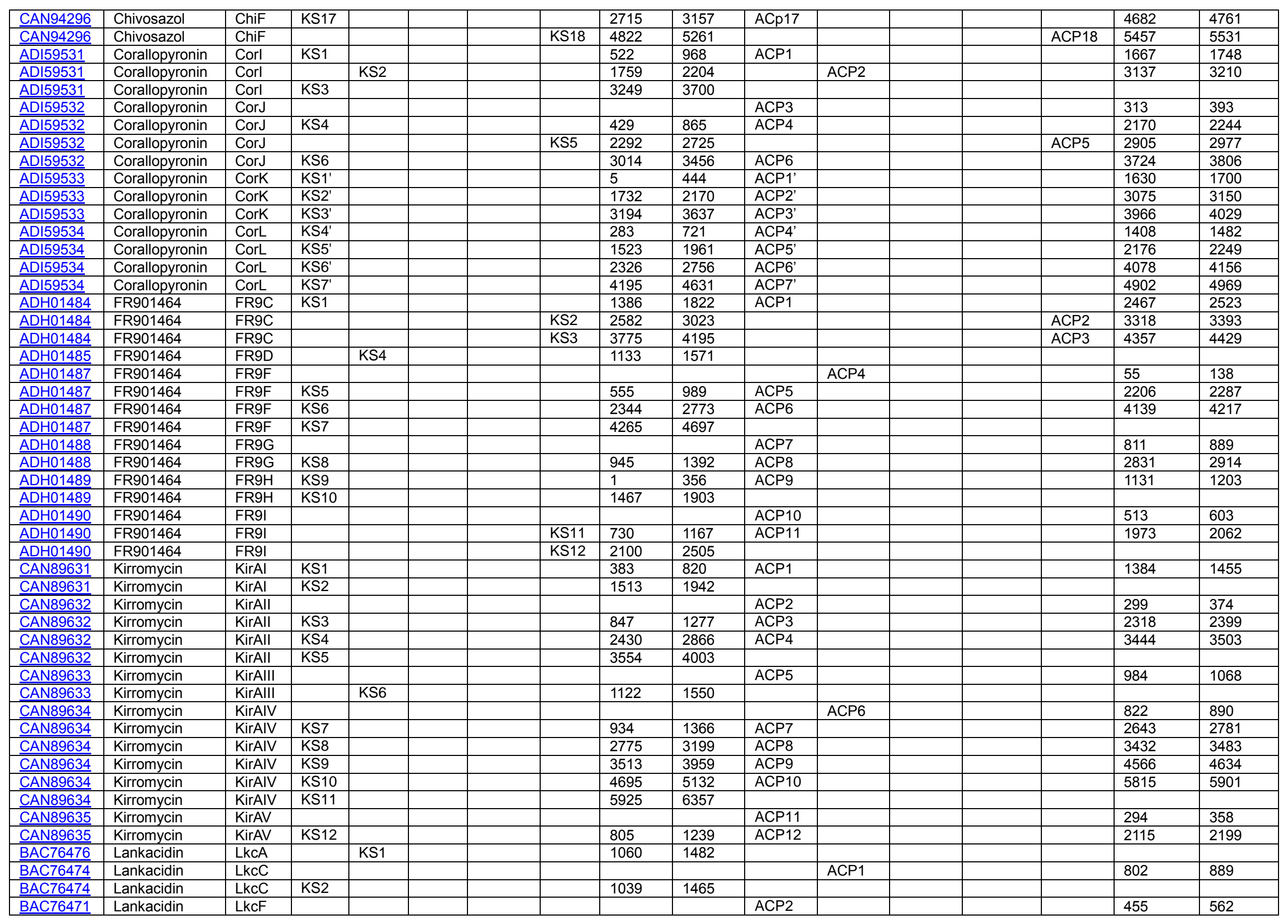




\begin{tabular}{|c|c|c|c|c|c|c|c|c|c|c|c|c|c|c|c|c|}
\hline BAC76471 & Lankacidin & LkcF & KS3 & & & & & 602 & 1029 & ACP3 & & & & & 1653 & 1728 \\
\hline$\overline{B A C 76471}$ & Lankacidin & LkcF & KS4 & & & & & 1780 & 2200 & & & & & & & \\
\hline BAC76470 & Lankacidin & LkcG & KS5 & & & & & 7 & 427 & ACP5 & & & & & 584 & 658 \\
\hline AAN85522 & Leinamycin & Lnml & & & KS1 & & & 1926 & 2355 & & & ACP1 & & & 2543 & 2618 \\
\hline AAN85522 & Leinamycin & Lnml & & & & KS2 & & 2659 & 3094 & & & & ACP2 & & 3822 & 3887 \\
\hline$\overline{\text { AAN85522 }}$ & Leinamycin & Lnml & KS3 & & & & & 3928 & 4361 & & & & & & & \\
\hline$\overline{\text { AAN85523 }}$ & Leinamycin & LnmJ & & & & & & & & ACP3 & & & & & 323 & 396 \\
\hline AAN85523 & Leinamycin & LnmJ & KS4 & & & & & 924 & 1359 & ACP4 & & & & & 1631 & 1709 \\
\hline AAN85523 & Leinamycin & LnmJ & KS5 & & & & & 1760 & 2198 & ACP5 & & & & & 3654 & 3719 \\
\hline$\overline{\text { AAN85523 }}$ & Leinamycin & LnmJ & KS6 & & & & & 3794 & 4241 & ACP6 & & & & & 5243 & 5316 \\
\hline AAN85523 & Leinamycin & LnmJ & KS7 & & & & & 5386 & 5827 & ACP7 & & & & & 6142 & 6220 \\
\hline$\overline{\text { AGS77289 }}$ & Myxopyronin & Mxnl & KS1 & & & & & 528 & 973 & ACP1 & & & & & 1695 & 1759 \\
\hline AGS77289 & Myxopyronin & Mxnl & & KS2 & & & & 1779 & 2224 & & ACP2 & & & & 3156 & 3237 \\
\hline AGS77289 & Myxopyronin & Mxnl & KS3 & & & & & 3276 & 3726 & & & & & & & \\
\hline$\overline{\text { AGS77290 }}$ & Myxopyronin & MxnJ & & & & & & & & ACP3 & & & & & 328 & 409 \\
\hline AGS77290 & Myxopyronin & MxnJ & KS4 & & & & & 448 & 884 & ACP4 & & & & & 2211 & 2276 \\
\hline AGS77290 & Myxopyronin & MxnJ & & & & & KS5 & 2320 & 2755 & & & & & ACP5 & 2930 & 3009 \\
\hline$\overline{\text { AGS77290 }}$ & Myxopyronin & MxnJ & KS6 & & & & & 3050 & 3492 & ACP6 & & & & & 3540 & 3601 \\
\hline$\overline{\text { AGS77291 }}$ & Myxopyronin & MxnK & KS1' & & & & & 14 & 453 & ACP1' & & & & & 1649 & 1724 \\
\hline AGS77291 & Myxopyronin & MxnK & & & & & KS2' & 1760 & 2198 & & & & & ACP2' & 2391 & 2470 \\
\hline AGS77291 & Myxopyronin & MxnK & 'KS3' & & & & & 2529 & 2967 & ACP3' & & & & & 3199 & 3273 \\
\hline AGS77291 & Myxopyronin & MxnK & KS4' & & & & & 3346 & 3776 & ACP4' & & & & & 5127 & 5204 \\
\hline$\overline{\text { AGS77291 }}$ & Myxopyronin & MxnK & KS5' & & & & & 5249 & 5685 & ACP5' & & & & & 5944 & 6025 \\
\hline$\overline{\mathrm{ABF} 85931}$ & Myxovirescin & Ta-1 & & KS1 & & & & 1087 & 1529 & & ACP1 & & & & 2132 & 2196 \\
\hline ABF85931 & Myxovirescin & Ta-1 & KS2 & & & & & 2235 & 2686 & ACP2 & & & & & 3584 & 3666 \\
\hline ABF85931 & Myxovirescin & Ta-1 & & & & & KS3 & 3694 & 4130 & & & & & ACP3 & 4317 & 4381 \\
\hline$\overline{\mathrm{ABF} 85931}$ & Myxovirescin & Ta-1 & KS4 & & & & & 4409 & 4840 & ACP4 & & & & & 5743 & 5820 \\
\hline ABF85931 & Myxovirescin & Ta-1 & KS5 & & & & & 5864 & 6314 & ACP5 & & & & & 6550 & 6622 \\
\hline ABF85931 & Myxovirescin & Ta-1 & KS6 & & & & & 6669 & 7102 & ACP6 & & & & & 8008 & 8093 \\
\hline ABF85931 & Myxovirescin & Ta-1 & KS7 & & & & & 8131 & 8583 & ACP7 & & & & & 8858 & 8939 \\
\hline$\overline{\mathrm{ABF}} 92489$ & Myxovirescin & $\mathrm{TaO}$ & KS8 & & & & & 451 & 885 & ACP8 & & & & & 1774 & 1865 \\
\hline ABF92489 & Myxovirescin & $\mathrm{TaO}$ & KS9 & & & & & 1915 & 2365 & ACP9 & & & & & 2855 & 2934 \\
\hline ABF92489 & Myxovirescin & $\mathrm{TaO}$ & KS10 & & & & & 2987 & 3437 & ACP10 & & & & & 4353 & 4433 \\
\hline ABF92489 & Myxovirescin & $\mathrm{TaO}$ & KS11 & & & & & 4499 & 4949 & & & & & & & \\
\hline$\overline{\mathrm{ABF} 88102}$ & Myxovirescin & TaP & & & & & & & & ACP11 & & & & & 327 & 453 \\
\hline$\overline{\mathrm{ABF} 88102}$ & Myxovirescin & TaP & KS12 & & & & & 499 & 931 & ACP12 & & & & & 2278 & 2350 \\
\hline ABF89568 & Myxovirescin & Tal & KS0 & & & & & 836 & 1274 & ACP0 & & & & & 2010 & 2082 \\
\hline ABF92824 & Myxovirescin & TaL & & & & & KSO' & 29 & 471 & & & & & ACPO' & 1330 & 1414 \\
\hline$\overline{\mathrm{ABF} 92824}$ & Myxovirescin & TaL & KS1' & & & & & 1438 & 1880 & ACP1' & & & & & 2056 & 2136 \\
\hline ADA69237 & Nosperin & NspA & KS1 & & & & & 581 & 1021 & ACP1 & & & & & 2146 & 2218 \\
\hline ADA69237 & Nosperin & NspA & KS2 & & & & & 2269 & 2720 & ACP2 & & & & & 3466 & 3546 \\
\hline ADA69237 & Nosperin & NspA & KS3 & & & & & 3741 & 4176 & ACP3 & & & & & 5163 & 5239 \\
\hline ADA69239 & Nosperin & $\mathrm{NspC}$ & & & & & KS4 & 35 & 476 & & & & & ACP4 & 646 & 729 \\
\hline$\overline{\text { ADA69239 }}$ & Nosperin & $\mathrm{NspC}$ & & KS5 & & & & 1827 & 2264 & & ACP5 & & & & 3632 & 3704 \\
\hline ADA69239 & Nosperin & NspC & & & & & KS6 & 3802 & 4244 & & & & & ACP6 & 4712 & 4778 \\
\hline ADA69239 & Nosperin & NspC & KS7 & & & & & 4818 & 5252 & ACP7 & & & & & 6320 & 6381 \\
\hline ADA69239 & Nosperin & NspC & & & & & KS8 & 6429 & 6861 & & & & & ACP8 & 7048 & 7127 \\
\hline$\overline{\text { ADA69241 }}$ & Nosperin & NspD & & KS9 & & & & 12 & 452 & & ACP9 & & & & 1836 & 1902 \\
\hline$\overline{A B S 90478}$ & Oxazolomycin & OzmQ & & KS1 & & & & 15 & 457 & & ACP1 & & & & 725 & 809 \\
\hline ABS90475 & Oxazolomycin & OzmN & KS2 & & & & & 15 & 454 & ACP2 & & & & & 1384 & 1470 \\
\hline ABS90475 & Oxazolomycin & OzmN & KS3 & & & & & 1522 & 1963 & ACP3 & & & & & 2943 & 3025 \\
\hline
\end{tabular}




\begin{tabular}{|c|c|c|c|c|c|c|c|c|c|c|c|c|c|c|c|c|}
\hline ABS90475 & Oxazolomycin & OzmN & KS4 & & & & & 3061 & 3513 & ACP4 & & & & & 4840 & 4915 \\
\hline$\overline{\mathrm{ABS} 90470}$ & Oxazolomycin & $\mathrm{OzmH}$ & KS5 & & & & & 32 & 481 & ACP5 & & & & & 1619 & 1690 \\
\hline ABS90470 & Oxazolomycin & $\mathrm{OzmH}$ & & KS6 & & & & 2761 & 3216 & & ACP6 & & & & 4107 & 4171 \\
\hline$\overline{A B S 90470}$ & Oxazolomycin & $\mathrm{OzmH}$ & KS7 & & & & & 4221 & 4663 & ACP7 & & & & & 6023 & 6078 \\
\hline ABS90470 & Oxazolomycin & $\mathrm{OzmH}$ & & & & & KS8 & 6118 & 6534 & & & & & & & \\
\hline ABS90470 & Oxazolomycin & $\mathrm{OzmH}$ & KS9 & & & & & 6548 & 6979 & ACP9 & & & & & 7590 & 7674 \\
\hline ABS90471 & Oxazolomycin & OzmJ & KS10 & & & & & 55 & 472 & ACP10 & & & & & 765 & 849 \\
\hline$\overline{\mathrm{ABS} 90471}$ & Oxazolomycin & OzmJ & & & & & KS11 & 909 & 1352 & & & & & ACP11 & 2590 & 2666 \\
\hline ABS90472 & Oxazolomycin & OzmK & KS12 & & & & & 80 & 506 & ACP12 & & & & & 1115 & 1185 \\
\hline$\overline{\text { AAR19304 }}$ & Pederin & Pedl & KS1 & & & & & 355 & 792 & ACP1 & & & & & 1836 & 1904 \\
\hline AAR19304 & Pederin & Pedl & KS2 & & & & & 1941 & 2393 & $\mathrm{ACP} 2$ & & & & & 3145 & 3228 \\
\hline$\overline{\text { AAR19304 }}$ & Pederin & Pedl & KS3 & & & & & 3399 & 3835 & ACP3 & & & & & 4788 & 4865 \\
\hline AAS47564 & Pederin & PedF & & & & & KS4 & 41 & 482 & & & & & ACP4 & 647 & 728 \\
\hline AAS47564 & Pederin & PedF & & KS5 & & & & 1838 & 2275 & & ACP5 & & & & 2912 & 2976 \\
\hline AAS47564 & Pederin & PedF & KS6 & & & & & 3023 & 3473 & ACP6 & & & & & 4550 & 4630 \\
\hline AAS47564 & Pederin & PedF & KS7 & & & & & 4674 & 5099 & ACP7 & & & & & 6327 & 6404 \\
\hline AAS47564 & Pederin & PedF & KS8 & & & & & 6458 & 6908 & ACP8 & & & & & 7580 & 7672 \\
\hline AAS47564 & Pederin & PedF & KS9 & & & & & 7722 & 8154 & & & & & & & \\
\hline$\overline{\text { AAS47562 }}$ & Pederin & PedH & & & & & & & & ACP9 & & & & & 65 & 134 \\
\hline AAS47562 & Pederin & PedH & KS10 & & & & & 218 & 665 & ACP10 & & & & & 1621 & 1700 \\
\hline AAS47562 & Pederin & PedH & KS11 & & & & & 1758 & 2208 & ACP11 & & & & & 2836 & 2918 \\
\hline AAS47562 & Pederin & PedH & KS12 & & & & & 2967 & 3419 & ACP12 & & & & & 4048 & 4125 \\
\hline AAS47562 & Pederin & PedH & & & & & KS13 & 4177 & 4623 & & & & & ACP13 & 4810 & 4892 \\
\hline ADA82581 & Psymberin & PsyA & KS1 & & & & & 728 & 1167 & ACP1 & & & & & 1788 & 1860 \\
\hline$\overline{\text { ADA82581 }}$ & Psymberin & PsyA & KS2 & & & & & 1898 & 2334 & ACP2 & & & & & 3230 & 3292 \\
\hline ADA82585 & Psymberin & PsyD & & & & & KS3 & 22 & 464 & & & & & ACP3 & 958 & 1023 \\
\hline ADA82585 & Psymberin & PsyD & & KS4 & & & & 2094 & 2531 & & ACP4 & & & & 3121 & 3197 \\
\hline ADA82585 & Psymberin & PsyD & KS5 & & & & & 3224 & 3671 & ACP5 & & & & & 4693 & 4774 \\
\hline ADA82585 & Psymberin & PsyD & KS6 & & & & & 4796 & 5221 & ACP6 & & & & & 6347 & 6428 \\
\hline ADA82585 & Psymberin & PsyD & KS7 & & & & & 6464 & 6911 & ACP7 & & & & & 7918 & 8003 \\
\hline ADA82585 & Psymberin & PsyD & KS8 & & & & & 8033 & 8443 & ACP8 & & & & & 9061 & 9128 \\
\hline ADA82585 & Psymberin & PsyD & KS9 & & & & & 9169 & 9592 & ACP9 & & & & & 10322 & 10396 \\
\hline ADA82585 & Psymberin & PsyD & KS10 & & & & & 10437 & 10877 & ACP10 & & & & & 11037 & 11139 \\
\hline ADA82585 & Psymberin & PsyD & KS11 & & & & & 11169 & 11607 & ACP11 & & & & & 12268 & 12347 \\
\hline CCA89326 & Rhizopodin & RizB & & KS1 & & & & 1558 & 2005 & & ACP1 & & & & 2901 & 2985 \\
\hline CCA89326 & Rhizopodin & RizB & KS2 & & & & & 3016 & 3458 & ACP2 & & & & & 4520 & 4585 \\
\hline$\overline{\overline{\text { CCA89326 }}}$ & Rhizopodin & RizB & & & & & KS3 & 4627 & 5064 & & & & & ACP3 & 5587 & 5651 \\
\hline CCA89326 & Rhizopodin & RizB & KS4 & & & & & 5691 & 6130 & ACP4 & & & & & 6314 & 6394 \\
\hline$\overline{\mathrm{CCA89326}}$ & Rhizopodin & RizB & KS5 & & & & & 6433 & 6860 & ACP5 & & & & & 8148 & 8231 \\
\hline CCA89326 & Rhizopodin & RizB & KS6 & & & & & 8623 & 9061 & ACP6 & & & & & 9722 & 9801 \\
\hline CCA89326 & Rhizopodin & RizB & & & & & KS7 & 9840 & 10264 & & & & & ACP7 & 10757 & 10827 \\
\hline CCA89326 & Rhizopodin & RizB & KS8 & & & & & 10854 & 11290 & & & & & & & \\
\hline CCA89327 & Rhizopodin & RizC & & & & & & & & ACP8 & & & & & 23 & 103 \\
\hline$\overline{\text { CCA89327 }}$ & Rhizopodin & RizC & KS9 & & & & & 148 & 586 & ACP9 & & & & & 1234 & 1312 \\
\hline$\overline{\text { CCA89327 }}$ & Rhizopodin & RizC & & & & & KS10 & 1351 & 1786 & & & & & ACP10 & 1962 & 2036 \\
\hline$\overline{\mathrm{CCA} 89328}$ & Rhizopodin & RizD & & & KS11 & & & 1781 & 2207 & & & ACP11 & & & 2384 & 2450 \\
\hline CCA89328 & Rhizopodin & RizD & & & & KS12 & & 2483 & 2922 & & & & ACP12 & & 3577 & 3642 \\
\hline$\overline{\text { CCA89328 }}$ & Rhizopodin & RizD & & & & & KS13 & 3673 & 4100 & & & & & ACP13 & 4586 & 4656 \\
\hline CCA89328 & Rhizopodin & RizD & KS14 & & & & & 4681 & 5118 & ACP14 & & & & & 5550 & 5622 \\
\hline$\overline{\mathrm{CCA89328}}$ & Rhizopodin & RizD & KS15 & & & & & 5666 & 6110 & ACP15 & & & & & 7059 & 7134 \\
\hline CCA89328 & Rhizopodin & RizD & KS16 & & & & & 7175 & 7615 & & & & & & & \\
\hline
\end{tabular}




\begin{tabular}{|c|c|c|c|c|c|c|c|c|c|c|c|c|c|c|c|c|}
\hline CCA89329 & Rhizopodin & RizE & & & & & & & & ACP16 & & & & & 540 & 618 \\
\hline$\overline{\text { CCA89329 }}$ & Rhizopodin & RizE & & & & & KS17 & 662 & 1106 & & & & & ACP17 & 1597 & 1671 \\
\hline CCA89329 & Rhizopodin & RizE & KS18 & & & & & 1708 & 2145 & ACP18 & & & & & 2798 & 2881 \\
\hline CCA89329 & Rhizopodin & RizE & & & & & KS19 & 2927 & 3366 & & & & & ACP19 & 3554 & 3642 \\
\hline CAL69888 & Rhizoxin & RhiA & KS1 & & & & & 783 & 1210 & ACP1 & & & & & 1378 & 1446 \\
\hline$\overline{\text { CAL69889 }}$ & Rhizoxin & RhiB & & & KS2 & & & 1036 & 1460 & & & ACP2 & & & 1641 & 1715 \\
\hline$\overline{\text { CAL69889 }}$ & Rhizoxin & RhiB & & & & KS3 & & 1752 & 2207 & & & & ACP3 & & 3625 & 3690 \\
\hline CAL69889 & Rhizoxin & RhiB & KS4 & & & & & 3748 & 4182 & ACP4 & & & & & 5067 & 5140 \\
\hline CAL69889 & Rhizoxin & RhiB & KS5 & & & & & 5285 & 5730 & & & & & & & \\
\hline$\overline{\text { CAL69890 }}$ & Rhizoxin & RhiC & & & & & & & & ACP5 & & & & & 386 & 456 \\
\hline$\overline{\text { CAL69890 }}$ & Rhizoxin & RhiC & KS6 & & & & & 517 & 941 & ACP6 & & & & & 2045 & 2119 \\
\hline CAL69890 & Rhizoxin & RhiC & KS7 & & & & & 2192 & 2617 & ACP7 & & & & & 3247 & 3325 \\
\hline CAL69890 & Rhizoxin & RhiC & KS8 & & & & & 3391 & 3834 & ACP8 & & & & & 5097 & 5170 \\
\hline CAL69891 & Rhizoxin & RhiD & KS9 & & & & & 56 & 482 & ACP9 & & & & & 1415 & 1495 \\
\hline$\overline{\text { CAL69891 }}$ & Rhizoxin & RhiD & KS10 & & & & & 1557 & 2007 & ACP10 & & & & & 3387 & 3461 \\
\hline CAL69891 & Rhizoxin & RhiD & & & & & KS11 & 3542 & 3981 & & & & & & & \\
\hline CAL69893 & Rhizoxin & RhiE & & & & & & & & & & & & ACP11 & 163 & 237 \\
\hline$\overline{C \text { CAL69893 }}$ & Rhizoxin & RhiE & KS12 & & & & & 295 & 730 & ACP12 & & & & & 1406 & 1470 \\
\hline$\overline{\text { CAL69893 }}$ & Rhizoxin & RhiE & KS13 & & & & & 1504 & 1934 & ACP13 & & & & & 2872 & 2950 \\
\hline CAL69893 & Rhizoxin & RhiE & KS14 & & & & & 3053 & 3502 & ACP14 & & & & & 4006 & 4084 \\
\hline CAL69894 & Rhizoxin & RhiF & KS15 & & & & & 53 & 484 & ACP15 & & & & & 1415 & 1840 \\
\hline CAL69894 & Rhizoxin & RhiF & & & & & KS16 & 1546 & 1991 & & & & & ACP16 & 2215 & 2278 \\
\hline$\overline{\mathrm{ABC} 34832}$ & Thailandamide & TaiD & KS1 & & & & & 11 & 459 & ACP1 & & & & & 1217 & 1286 \\
\hline ABC34832 & Thailandamide & TaiD & KS2 & & & & & 1371 & 1792 & ACP2 & & & & & 2848 & 2935 \\
\hline ABC34832 & Thailandamide & TaiD & KS3 & & & & & 3003 & 3441 & ACP3 & & & & & 4833 & 4914 \\
\hline ABC34832 & Thailandamide & TaiD & & & & & KS4 & 4996 & 5435 & & & & & ACP4 & 5464 & 5544 \\
\hline$\overline{A B C 35522}$ & Thailandamide & TaiE & & KS5 & & & & 1235 & 1678 & & ACP5 & & & & 2663 & 2730 \\
\hline ABC35522 & Thailandamide & TaiE & KS6 & & & & & 2845 & 3297 & ACP6 & & & & & 3762 & 3893 \\
\hline$\overline{\mathrm{ABC} 34176}$ & Thailandamide & TaiK & KS7 & & & & & 74 & 510 & ACP7 & & & & & 1267 & 1339 \\
\hline ABC34176 & Thailandamide & TaiK & & & & & KS8 & 1450 & 1869 & & & & & & & \\
\hline$\overline{\mathrm{ABC} 34675}$ & Thailandamide & TaiL & & & & & & & & & & & & ACP8 & 361 & 441 \\
\hline ABC34675 & Thailandamide & TaiL & KS9 & & & & & 509 & 958 & ACP9 & & & & & 1262 & 1322 \\
\hline ABC34675 & Thailandamide & TaiL & KS10 & & & & & 1374 & 1821 & ACP10 & & & & & 2536 & 2619 \\
\hline ABC34675 & Thailandamide & TaiL & KS11 & & & & & 2751 & 3199 & ACP11 & & & & & 4254 & 4334 \\
\hline$\overline{\mathrm{ABC} 34675}$ & Thailandamide & TaiL & KS12 & & & & & 4463 & 4910 & ACP12 & & & & & 5384 & 5451 \\
\hline$\overline{A B C 33986}$ & Thailandamide & TaiM & KS13 & & & & & 361 & 797 & ACP13 & & & & & 1689 & 1758 \\
\hline$\overline{A B C 33986}$ & Thailandamide & TaiM & KS14 & & & & & 1872 & 2316 & ACP14 & & & & & 3713 & 3789 \\
\hline ABC33986 & Thailandamide & TaiM & KS15 & & & & & 3891 & 4310 & & & & & & & \\
\hline$\overline{\mathrm{ABC} 34599}$ & Thailandamide & TaiN & & & & & & & & ACP15 & & & & & 472 & 536 \\
\hline$\overline{A B C 34599}$ & Thailandamide & TaiN & & & & & KS16 & 656 & 1088 & & & & & ACP16 & 1683 & 1739 \\
\hline AGN11880 & Thailanstatin & TstC & KS1 & & & & & 1271 & 1707 & ACP1 & & & & & 2339 & 2403 \\
\hline AGN11880 & Thailanstatin & TstC & & & & & KS2 & 2454 & 2895 & & & & & ACP2 & 3197 & 3279 \\
\hline AGN11880 & Thailanstatin & TstC & & & & & KS3 & 3700 & 4121 & & & & & ACP3 & 4285 & 4365 \\
\hline AGN11881 & Thailanstatin & $\begin{array}{l}\text { TstDE } \\
\mathrm{F}\end{array}$ & & KS4 & & & & 1122 & 1560 & & ACP4 & & & & 2929 & 3023 \\
\hline AGN11881 & Thailanstatin & $\begin{array}{l}\text { TstDE } \\
\text { F }\end{array}$ & KS5 & & & & & 3560 & 3994 & ACP5 & & & & & 5228 & 5309 \\
\hline AGN11881 & Thailanstatin & $\begin{array}{l}\text { TstDE } \\
\mathrm{F}\end{array}$ & KS6 & & & & & 5462 & 5891 & ACP6 & & & & & 7268 & 7350 \\
\hline$\underline{\text { AGN11881 }}$ & Thailanstatin & $\begin{array}{l}\text { TstDE } \\
F\end{array}$ & KS7 & & & & & 7399 & 7828 & & & & & & & \\
\hline
\end{tabular}




\begin{tabular}{|c|c|c|c|c|c|c|c|c|c|c|c|c|c|c|c|c|}
\hline AGN11882 & Thailanstatin & TstGH & & & & & & & & ACP7 & & & & & 845 & 911 \\
\hline AGN11882 & Thailanstatin & TstGH & KS8 & & & & & 977 & 1424 & ACP8 & & & & & 2070 & 2153 \\
\hline AGN11882 & Thailanstatin & TstGH & KS9 & & & & & 2202 & 2646 & ACP9 & & & & & 3380 & 3457 \\
\hline AGN11882 & Thailanstatin & TstGH & KS10 & & & & & 3646 & 4082 & & & & & & & \\
\hline$\overline{\text { AGN11883 }}$ & Thailanstatin & Tstl & & & & & & & & ACP10 & & & & & 542 & 623 \\
\hline AGN11883 & Thailanstatin & Tstl & & & & & KS11 & 744 & 1181 & ACP11 & & & & & 2002 & 2075 \\
\hline AGN11883 & Thailanstatin & Tstl & & & & & KS12 & 2127 & 2533 & & & & & & & \\
\hline$\overline{\text { BAF50727 }}$ & Virginiamycin & VirA & KS1 & & & & & 13 & 452 & ACP1 & & & & & 1693 & 1760 \\
\hline$\overline{\text { BAF50727 }}$ & Virginiamycin & VirA & KS2 & & & & & 1926 & 2373 & ACP2 & & & & & 3348 & 3422 \\
\hline BAF50727 & Virginiamycin & VirA & & KS3 & & & & 4549 & 4980 & & ACP3 & & & & 5873 & 5949 \\
\hline BAF50727 & Virginiamycin & VirA & KS4 & & & & & 6000 & 6448 & ACP4 & & & & & 6734 & 6810 \\
\hline BAF50722 & Virginiamycin & VirF & KS5 & & & & & 1 & 404 & & & & & & & \\
\hline$\overline{\text { BAF50721 }}$ & Virginiamycin & VirG & & & & & & & & ACP5 & & & & & 338 & 411 \\
\hline$\overline{\text { BAF50721 }}$ & Virginiamycin & VirG & KS6 & & & & & 473 & 909 & ACP6 & & & & & 1129 & 1203 \\
\hline AGN74892 & Griseoviridin & SgvE1 & KS1 & & & & & 568 & 980 & ACP1 & & & & & 1652 & 1731 \\
\hline AGN74892 & Griseoviridin & SgvE1 & KS2 & & & & & 1776 & 2200 & ACP2 & & & & & 3272 & 3347 \\
\hline$\overline{\text { AGN74893 }}$ & Griseoviridin & SgvE2 & & KS3 & & & & 18 & 433 & & ACP3 & & & & 1379 & 1458 \\
\hline$\overline{\text { AGN74893 }}$ & Griseoviridin & SgvE2 & KS4 & & & & & 1514 & 1951 & & & & & & & \\
\hline AGN74894 & Griseoviridin & SgvE3 & KS5 & & & & & 78 & 501 & ACP5 & & & & & 1253 & 1304 \\
\hline$\overline{\text { AGN74894 }}$ & Griseoviridin & SgvE3 & KS6 & & & & & 1394 & 1818 & ACP6 & & & & & 2070 & 2154 \\
\hline $\begin{array}{l}\text { CBW4574 } \\
9\end{array}$ & Pristinamycin & SnaE1 & KS1 & & & & & 581 & 1000 & ACP1 & & & & & 2309 & 2388 \\
\hline$\underline{\underline{9}}$ & Pristinamycin & SnaE1 & KS2 & & & & & 2561 & 2967 & ACP2 & & & & & 3953 & 4025 \\
\hline$\frac{\bar{C}}{8}$ & Pristinamycin & SnaE2 & & KS3 & & & & 17 & 433 & & ACP3 & & & & 1376 & 1447 \\
\hline $\begin{array}{l}\overline{\text { CBW4574 }} \\
\underline{8}\end{array}$ & Pristinamycin & SnaE2 & KS4 & & & & & 1536 & 1965 & ACP4 & & & & & 2289 & 2350 \\
\hline$\frac{\bar{C} \text { CBW4574 }}{1}$ & Pristinamycin & SnaE3 & KS5 & & & & & 37 & 463 & ACP5 & & & & & 1106 & 1186 \\
\hline $\begin{array}{l}\text { CBW4574 } \\
1\end{array}$ & Pristinamycin & SnaE3 & KS6 & & & & & 1239 & 1662 & ACP6 & & & & & 1910 & 1987 \\
\hline AHC73539 & Patellazoles & PtzF & KS1 & & & & & 4 & 419 & ACP1 & & & & & 1704 & 1782 \\
\hline $\mathrm{AHC73539}$ & Patellazoles & PtzF & & & & & KS2 & 1832 & 2218 & & & & & ACP2 & 2371 & 2427 \\
\hline AHC73998 & Patellazoles & PtzA & & & KS3 & & & 19 & 432 & & & ACP3 & & & 603 & 670 \\
\hline$\overline{\mathrm{AHC73998}}$ & Patellazoles & PtzA & & & & KS4 & & 710 & 1134 & & & & ACP4 & & 2424 & 2491 \\
\hline $\mathrm{AHC73998}$ & Patellazoles & PtzA & KS5 & & & & & 2524 & 2945 & & & & & & & \\
\hline$\overline{\mathrm{AHC73991}}$ & Patellazoles & PtzE & & & & & & & & ACP5 & & & & & 365 & 433 \\
\hline $\mathrm{AHC73991}$ & Patellazoles & PtzE & KS6 & & & & & 462 & 871 & ACP6 & & & & & 1743 & 1808 \\
\hline$\overline{\mathrm{AHC73991}}$ & Patellazoles & PtzE & KS7 & & & & & 1853 & 2273 & ACP7 & & & & & 3502 & 3575 \\
\hline AHC73991 & Patellazoles & PtzE & KS8 & & & & & 3615 & 4033 & ACP8 & & & & & 4695 & 4760 \\
\hline $\mathrm{AHC73997}$ & Patellazoles & PtzB & & & & & KS9 & 404 & 807 & & & & & & & \\
\hline $\mathrm{AHC73996}$ & Patellazoles & PtzC & & & & & & & & & & & & ACP9 & 31 & 104 \\
\hline$\overline{\mathrm{AHC73996}}$ & Patellazoles & PtzC & KS10 & & & & & 128 & 542 & ACP10 & & & & & 1780 & 1848 \\
\hline $\mathrm{AHC73996}$ & Patellazoles & PtzC & KS11 & & & & & 1892 & 2308 & ACP11 & & & & & 3121 & 3202 \\
\hline AHC73996 & Patellazoles & PtzC & KS12 & & & & & 3250 & 3673 & ACP12 & & & & & 4856 & 4926 \\
\hline $\mathrm{AHC73995}$ & Patellazoles & PtzD & KS13 & & & & & 58 & $58-468$ & ACP13 & & & & & 1007 & 1075 \\
\hline $\mathrm{AHC73995}$ & Patellazoles & PtzD & KS14 & & & & & 1106 & 1524 & ACP14 & & & & & 2859 & 2936 \\
\hline $\mathrm{AHC73995}$ & Patellazoles & PtzD & KS15 & & & & & 2232 & 2646 & ACP15 & & & & & 2956 & 3038 \\
\hline$\overline{\mathrm{AHC73995}}$ & Patellazoles & PtzD & KS16 & & & & & 3288 & 3710 & ACP16 & & & & & 4953 & 5023 \\
\hline $\mathrm{AHC73995}$ & Patellazoles & PtzD & & & & & KS17 & 5089 & 5498 & & & & & ACP17 & 6034 & 6100 \\
\hline
\end{tabular}


Figure S1. Phylogenetic tree of the KS domains from the selected 23 NRPS-AT-less type I PKS machinery (Table S3). Bootstrap consensus tree was generated by MEGA6 using the maximum likelihood method with a bootstrap test of 100 replicates. The proposed normal function KSs at the interface ( $\mathrm{KSi}$, Table S3) were highlighted in orange. Five of the seven proposed non-elongating KSs at the interface of NRPS-PKS (KSi0, Table S3) contained the mutated catalytic triad (represented by C2090-A2225-H2264 in Lnml-KS1) were highlighted in red, while the remaining two KSs CalA-KS1 and PtzA-KS3 without the mutated catalytic triad were in green. The KSi0+1 (Table S3) were highlighted in blue. KASIl (accession number: CAQ31616) was used as the outgroup.

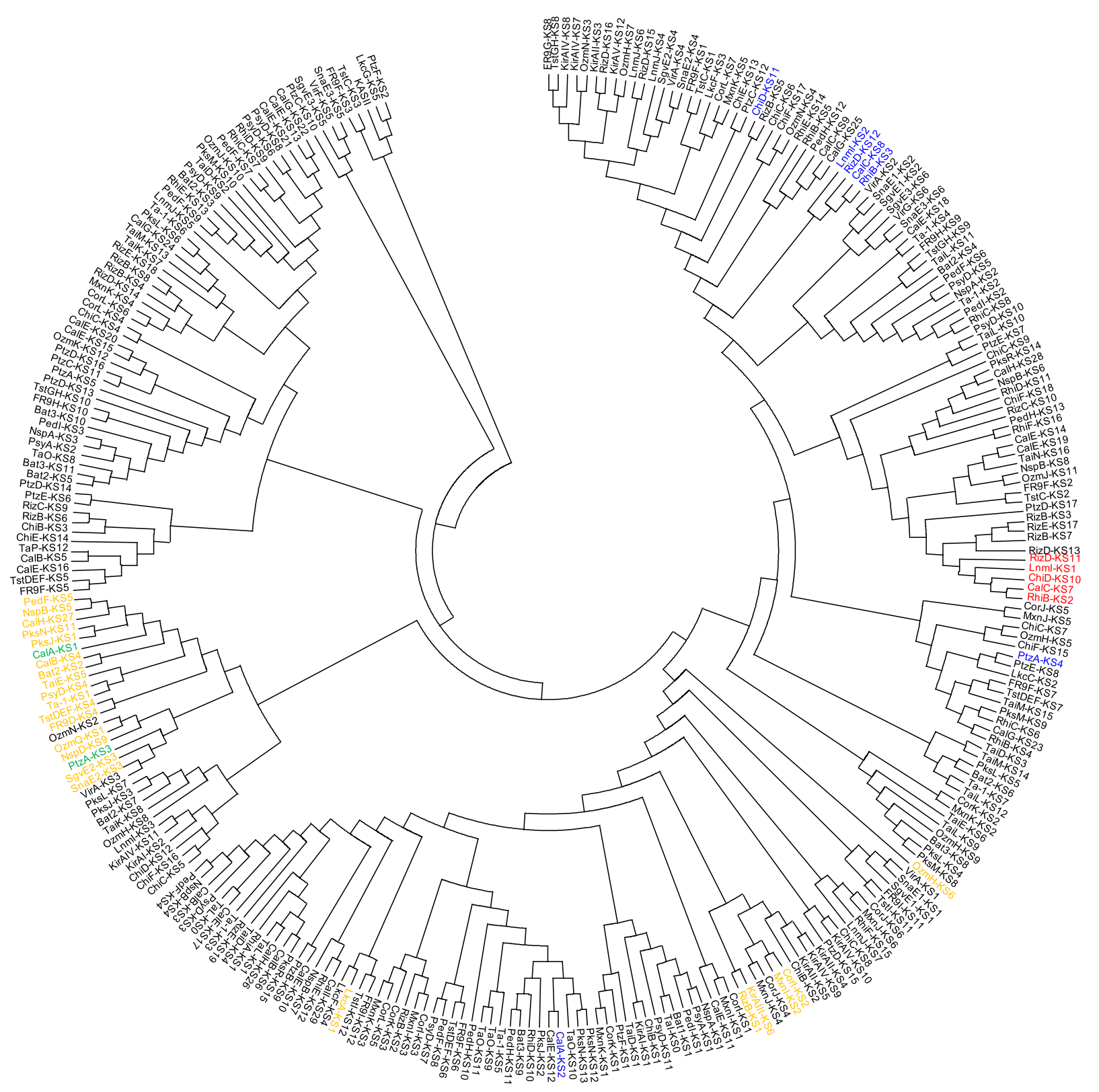


Figure S2. Sequence alignment of selected KS domains at the NRPS-AT-less type I PKS interfaces (Table S3). Proposed non-elongating KSs: Lnml-KS1, RizD-KS11, ChiD-KS10, CalC-KS7, and RhiB-KS2. Proposed normal function KSs: PksJ-KS1, CalB-KS4, FR9D-KS4, OzmQ-KS1, and TaiE-KS5. The mutated catalytic triad C2090-A2225-H2264 (number according to Lnml protein sequence) for the non-elongating KSs are highlighted and marked.
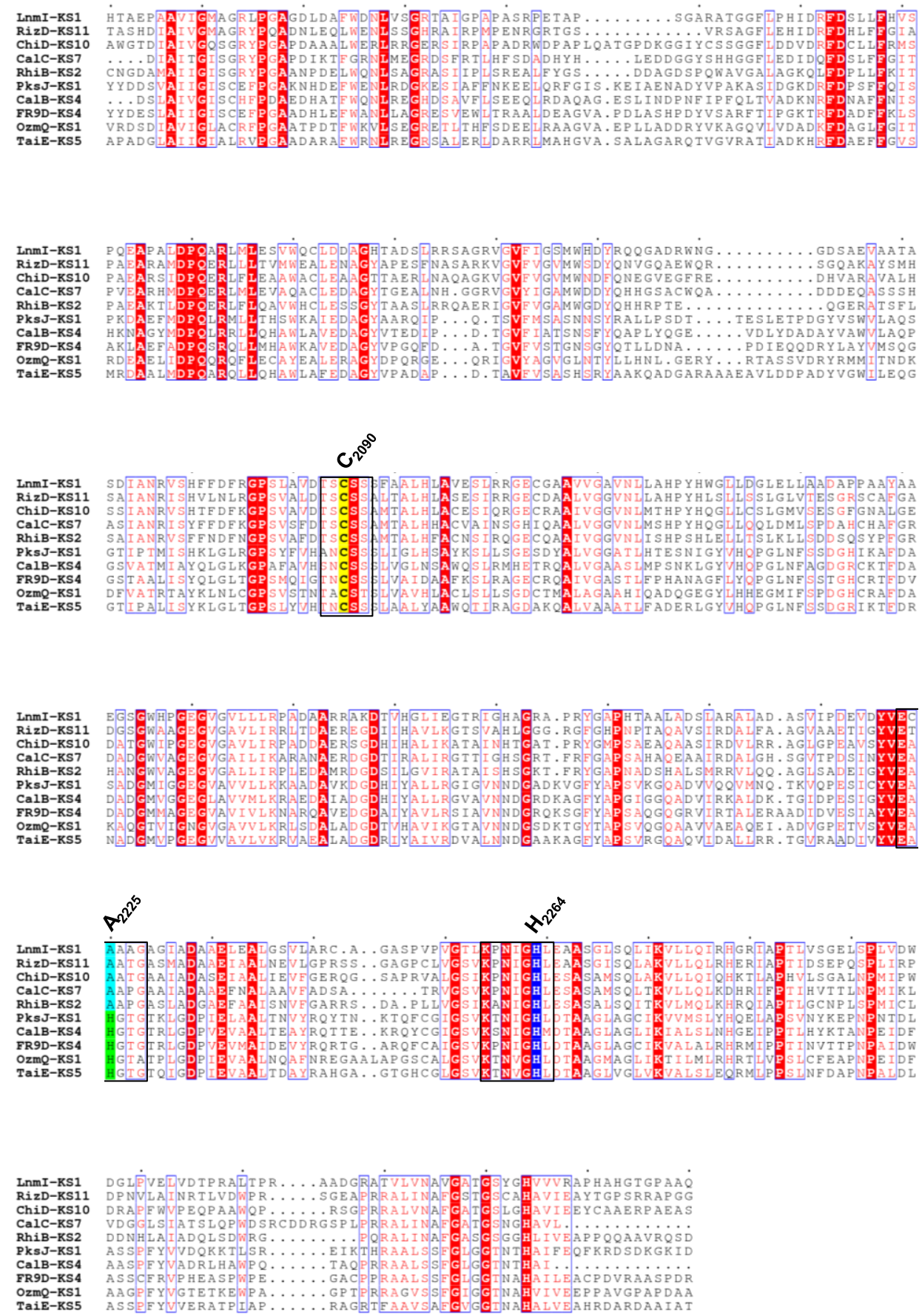
Figure S3. Phylogenetic tree of the ACP domains from the selected 23 NRPS-AT-less type I PKS machinery (Table S3). Bootstrap consensus tree was generated by MEGA6 using the maximum likelihood method with a bootstrap test of 100 replicates. ACPi (Table S3) were highlighted in orange, while ACPi0 (Table S3) were highlighted in red. The KSi0+1 (Table S3) were highlighted in blue. The thioesterase domain of LnmJ (LnmJ-TE, accession number: AAN85523) was used as the outgroup.

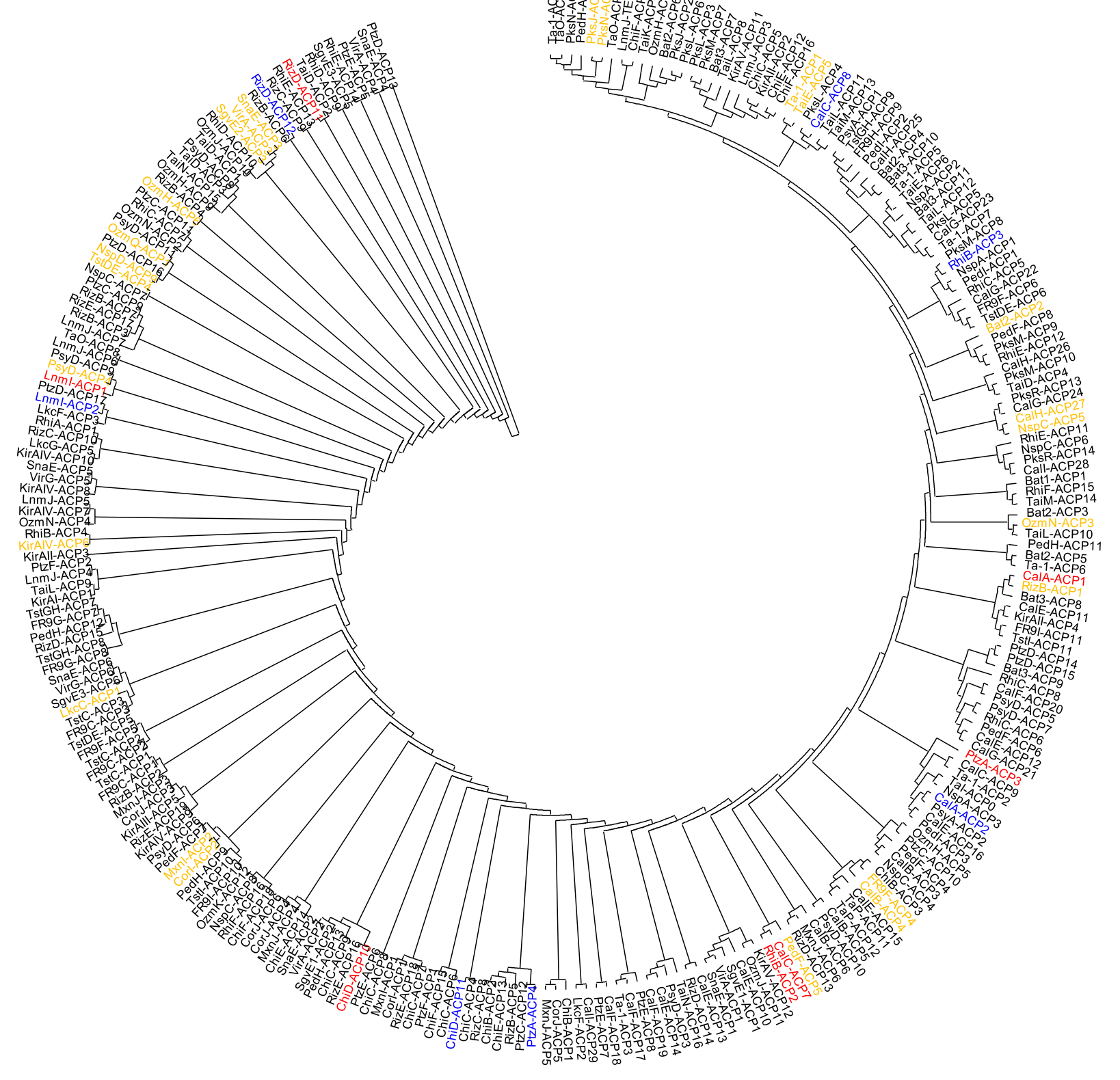


Figure S4. Generation of $I n m I-K S 1, I n m l-K S 2$, and $I n m l-A C P 1$ point mutation strains (A) and their southern analysis (B, C, D, E). A. Strategy for generation of Lnml-KS1, Lnml-KS2, and Lnml-ACP1 point mutation strains (generation of strain SB3036 with point mutation C2090A in Lnml-KS1 was shown). Southern analysis of the mutant strains SB3035, SB3036 (C2090A), SB3037 (H2959A), and SB3038 (C2824A), showing the expected fragment sizes upon BamHI digestion with (B) probe P1 (P1 was the $1.0 \mathrm{~kb}$ Xhol-Ncol fragment containing partial Inml-Ox and Inml-KS1 from pBS3112) and (C) probe P2 (P2 was the 1.5-kb apramycin resistance gene aac(3)IV from pUO9090). Lane 1, SB3035; lane 2-4, SB3036, SB3037, and SB3038; lane 5, S. atroolivaceous S-140 wild-type; M, molecular weight marker. Southern analysis of the mutant strains SB3039 $\left(D_{2580} S_{2581}-D_{2599} S_{2600}\right)$, SB3040 $\left(A_{2580} A_{2581}-D_{2599} S_{2600}\right)$, SB3041 $\left(A_{2580} A_{2581}\right.$ $\left.\mathrm{A}_{2599} \mathrm{~A}_{2600}\right)$, SB3042 $\left(\mathrm{A}_{2580} \mathrm{~A}_{2581}-\mathrm{S}_{2599} \mathrm{~S}_{2600}\right), \quad \mathrm{SB} 3043 \quad\left(\mathrm{~S}_{2580} \mathrm{~S}_{2581}-\mathrm{A}_{2599} \mathrm{~A}_{2600}\right)$, and SB3044 $\left(D_{2580} S_{2581}-A_{2599} A_{2600}\right)$, showing the expected fragment sizes upon BamHI digestion using (D) probe P1 and (E) probe P2. Lane 1, SB3035; lane 2-7, SB3039, SB3040, SB3041, SB3042, SB3043, and SB3044; lane 8, S. atroolivaceous S-140 wild-type; M, molecular weight marker. B1-B4, different BamHI sites; N, Ncol; X, Xhol. Apr, apramycin; Thi, thiostrepton; tsr, thiostrepton resistance gene.

A
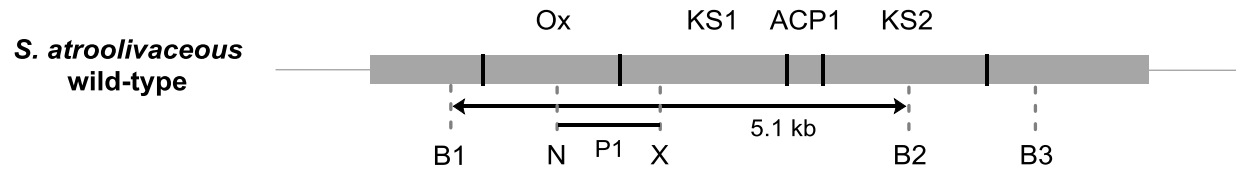

B3035

$\left(\mathrm{Apr}^{\mathrm{R}}\right)$

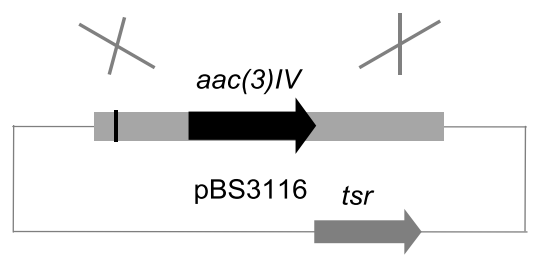

$\sqrt{ }$ Double crossover

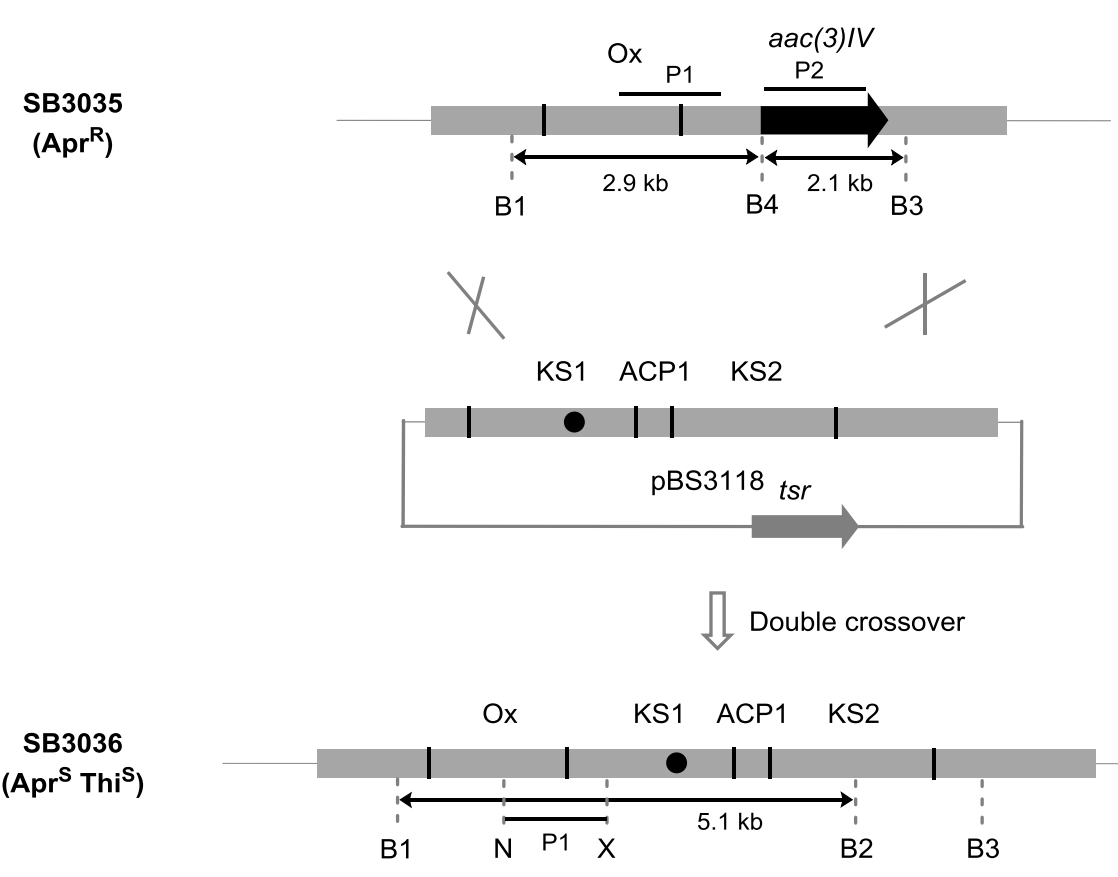



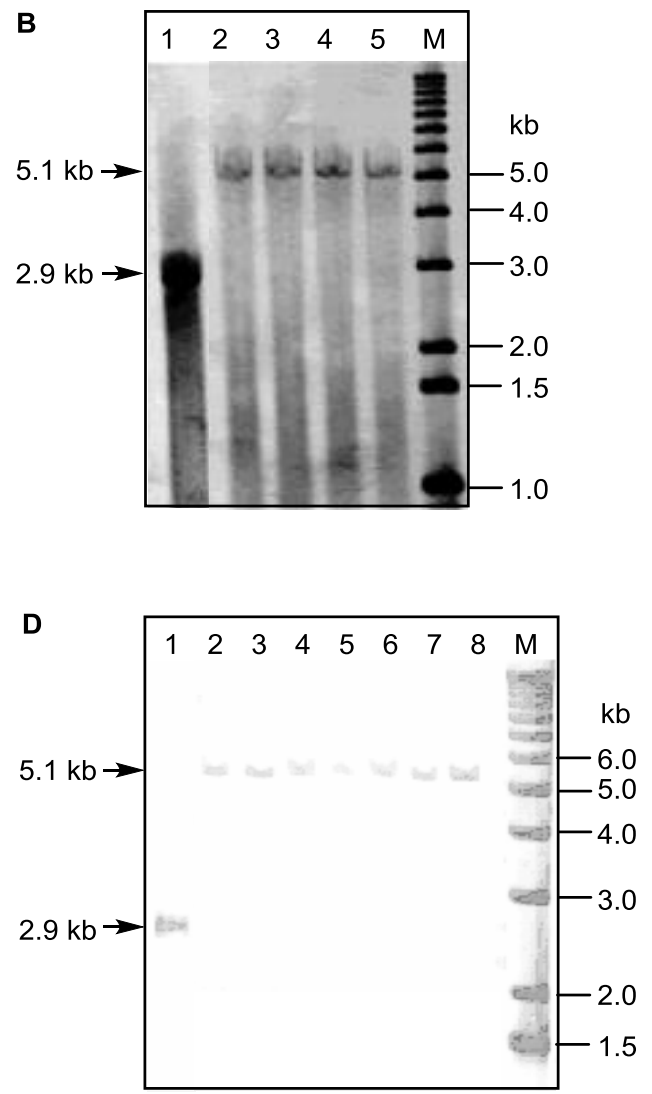
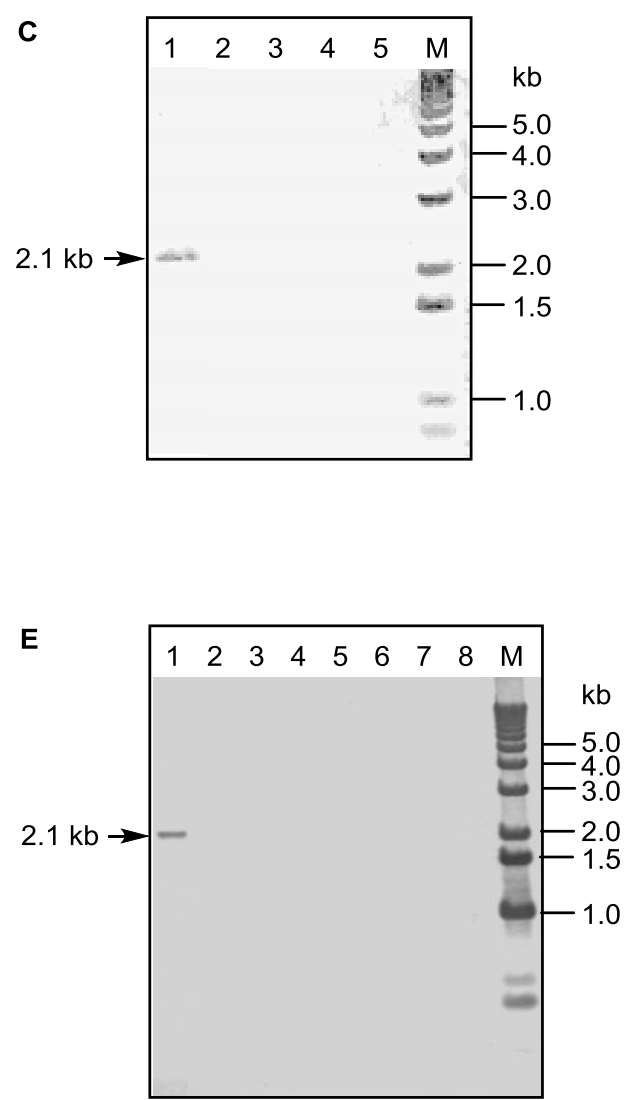